\title{
LARGE DEVIATIONS FOR OCCUPATION TIME PROFILES OF RANDOM INTERLACEMENTS
}

\author{
Xinyi Li and Alain-Sol Sznitman
}

\begin{abstract}
We derive a large deviation principle for the density profile of occupation times of random interlacements at a fixed level in a large box of $\mathbb{Z}^{d}, d \geq 3$. As an application, we analyze the asymptotic behavior of the probability that atypically high values of the density profile insulate a macroscopic body in a large box. As a step in this program, we obtain a similar large deviation principle for the occupation-time measure of Brownian interlacements at a fixed level in a large box of $\mathbb{R}^{d}$, and we derive a new identity for the Laplace transform of the occupation-time measure, which is based on the analysis of certain Schrödinger semi-groups.
\end{abstract}

Departement Mathematik ETH Zürich

CH-8092 Zürich

Switzerland

This research was supported in part by the grant ERC-2009-AdG 245728-RWPERCRI 



\section{Introduction}

Random interlacements have been instrumental in the analysis of various questions concerning the disconnection or the fragmentation created by random walk trajectories, see [5], [27], [32]. The existence of a non-trivial phase transition for the percolative properties of the vacant set of random interlacements, when one increases the level $u$ of the interlacement, plays an important role in their analysis. As it turns out, the level $u$ of random interlacements can also be measured by means of the random field of occupation times, which, in the case of $\mathbb{Z}^{d}, d \geq 3$, is stationary, ergodic, and has average value $u$. In this work, we study the large deviations of the density profile of this random field in a large box of $\mathbb{Z}^{d}$. As an application of the general large deviation principle we obtain, we analyze the asymptotic behavior of the probability that atypically high values of the density profile insulate a macroscopic body in a large box. One may naturally wonder whether such type of large deviations of the occupation-time profile actually captures the main mechanism for an atypical disconnection of a macroscopic body from infinity by the random interlacements, when the vacant set is in a percolative regime. In the course of our program, we derive a similar large deviation principle for the occupation-time measure of Brownian interlacements at a fixed level, in a large box of $\mathbb{R}^{d}, d \geq 3$. The scaling invariance of Brownian interlacements permits to recast this problem in terms of general Cramér theory, and our results rely on a new identity for the Laplace transform of the occupation-time measure, which is based on the analysis of Schrödinger semi-groups.

We now discuss our results in more detail. We consider continuous time random interlacements on $\mathbb{Z}^{d}, d \geq 3$. In essence, this is a Poisson point process on a certain state space consisting of doubly infinite $\mathbb{Z}^{d}$-valued trajectories marked by their duration at each step, modulo time-shift. A non-negative parameter $u$ comes as a multiplicative factor of the intensity measure of this Poisson point process, which is defined on a certain canonical space, see [29], which we denote here by $(\bar{\Omega}, \overline{\mathcal{A}}, \overline{\mathbb{P}})$. The field of occupation times of random interlacements at level $u$ is denoted by $L_{x, u}(\omega)$, for $x \in \mathbb{Z}^{d}, u \geq 0, \omega \in \bar{\Omega}$. It records the total duration spent at $x$ by the trajectories modulo time-shift with label at most $u$ in the cloud $\omega$, see [29].

Given a closed box $B \subseteq \mathbb{R}^{d}$ (by this we mean that $B$ is the product of $d$ non-degenerate compact intervals in $\mathbb{R}$ ), a central object of interest in this work is the density profile of the occupation times at level $u$ in the large discrete box $(N B) \cap \mathbb{Z}^{d}$ :

$$
\rho_{N, u}=\frac{1}{N^{d}} \sum_{x \in(N B) \cap \mathbb{Z}^{d}} L_{x, u} \delta_{\frac{x}{N}}, \text { for } N \geq 1, u \geq 0 .
$$

We view $\rho_{N, u}$ as a random element of $M_{+}(B)$, the set of positive measures on $B$, which we endow with the weak topology generated by $C(B)$, the set of continuous functions on $B$, and with its corresponding Borel $\sigma$-algebra. As a consequence of the ergodic theorem, see (4.4), $\overline{\mathbb{P}}$-a.s., $\rho_{N, u}$ converges weakly to $u m_{B}$, where $m_{B}$ stands for the restriction of the Lebesgue measure to Borel subsets of $B$. In Theorem 5.8, we establish a large deviation principle for $\rho_{N, u}$, which shows that for $u>0$,

as $N \rightarrow \infty$, the laws of $\rho_{N, u}$ on $M_{+}(B)$ satisfy a large deviation principle at speed $N^{d-2}$ with the convex, good rate function $\frac{1}{d} I_{u}$, 
where for $v>0$ and $\mu \in M_{+}(B)$, we have defined

$$
I_{v}(\mu)=\left\{\begin{array}{cl}
+\infty, & \text { if } \mu \text { is not absolutely continuous with respect to } m_{B}, \\
\inf \left\{\frac{1}{2} \int_{\mathbb{R}^{d}}|\nabla \varphi|^{2} d y ; \varphi \in H^{1}\left(\mathbb{R}^{d}\right), \varphi=\sqrt{\frac{d \mu}{d m_{B}}}-\sqrt{v}, \text { a.e. on } B\right\} \\
\text { if } \mu \text { is absolutely continuous with respect to } m_{B} \text { (and the } \\
\text { infimum of the empty set equals }+\infty) .
\end{array}\right.
$$

In other words, $I_{v}$ is a non-negative, convex, lower semi-continuous function, with compact level sets $\left\{I_{v} \leq a\right\}$, for $a \geq 0$, and for any open subset $O$ and closed subset $C$ of $M_{+}(B)$, we have

$$
\begin{aligned}
& \text { i) } \quad \underset{N}{\limsup } \frac{1}{N^{d-2}} \log \overline{\mathbb{P}}\left[\rho_{N, u} \in C\right] \leq-\inf _{C} \frac{1}{d} I_{u} \text {, and } \\
& \text { ii) } \quad \liminf _{N} \frac{1}{N^{d-2}} \log \overline{\mathbb{P}}\left[\rho_{N, u} \in O\right] \geq-\inf _{O} \frac{1}{d} I_{u} .
\end{aligned}
$$

In the course of proving Theorem 5.8, we derive a large deviation principle for the density profile of the occupation-time measure of Brownian interlacements at level $\alpha>0$ in a large box $L B$ of $\mathbb{R}^{d}, d \geq 3$, which is of independent interest. Letting $\mathcal{L}_{\alpha}$ stand for the random Radon measure of occupation times in $\mathbb{R}^{d}$ of Brownian interlacements at level $\alpha$, see (1.23) (or Section 2 of [31]) we consider in place of $\rho_{N, u}$ the random measure $\nu_{L, \alpha}$ on $B$, such that for any bounded measurable $f$ on $B$, the integral $\left\langle\nu_{L, \alpha}, f\right\rangle$ equals

$$
\left\langle\nu_{L, \alpha}, f\right\rangle=\frac{1}{L^{d}} \int_{L B} f\left(\frac{y}{L}\right) \mathcal{L}_{\alpha}(d y), \text { for } L \geq 1, \alpha \geq 0
$$

We show in Corollary 3.3 that for $\alpha>0$,

$$
\text { as } L \rightarrow \infty \text {, the laws of } \nu_{L, \alpha} \text { on } M_{+}(B) \text { satisfy a large deviation principle, }
$$
at speed $L^{d-2}$ with the convex good rate function $I_{\alpha}$ (see $(0.3)$ ).

As an application of the large deviation principle (0.2), we analyze the asymptotic behavior of the probability that high values of the (smoothed-out) density profile insulate a large macroscopic body. We consider a regularization $f_{N}$ of $\rho_{N, u}$ obtained by the convolution of $\rho_{N, u}$ with a continuous probability density $\varphi_{\delta}$ supported in the closed Euclidean ball of radius $\delta$ centered at the origin of $\mathbb{R}^{d}$. Given a compact subset $K$ of a sub-box $B_{0}$ of $B$ (at distance at least $\delta$ from $B^{c}$ ), and a positive number $a$, we are interested in the event $\left\{\rho_{N, u} \in \mathcal{D}_{a, \delta}\right\}$ (see (6.5) for the precise definition), where the level set $\left\{f_{N} \geq a\right\}$ "disconnects $K$ from $\partial B_{0}$ ". We show in Theorems 6.2 and 6.4 that when $a>u$,

$$
\begin{aligned}
\text { i) } \quad \limsup _{N} \frac{1}{N^{d-2}} \log \overline{\mathbb{P}}\left[\rho_{N, u} \in \mathcal{D}_{a, \delta}\right] \leq-\frac{1}{d}(\sqrt{a}-\sqrt{u})^{2} \operatorname{cap}(K), \\
\text { ii) } \quad \liminf _{N} \frac{1}{N^{d-2}} \log \overline{\mathbb{P}}\left[\rho_{N, u} \in \mathcal{D}_{a, \delta}\right] \geq-\frac{1}{d}(\sqrt{a}-\sqrt{u})^{2} \operatorname{cap}\left(K^{\delta}\right),
\end{aligned}
$$

where $\operatorname{cap}(\cdot)$ stands for the Brownian capacity (see below (1.21)) and $K^{\delta}$ for the closed $\delta$-neighborhood of $K$. As $\delta \rightarrow 0$, one knows that $\operatorname{cap}\left(K^{\delta}\right) \downarrow \operatorname{cap}(K)$, see Remark 6.51 ), so that upper and lower bounds in (0.7) become identical. Actually, one can let $\delta$ slowly 
tend to 0 so that the corresponding upper and lower bounds match with the right-hand side of (0.7) i), see Remark 6.5 2).

The asymptotics (0.7) has an interesting consequence. There is an intuitive strategy to ensure that the level set $\left\{f_{N} \geq a\right\}$ disconnects $K$ from $\partial B_{0}$ (or in other words that $\left\{\rho_{N, u} \in \mathcal{D}_{a, \delta}\right\}$ occurs). Roughly speaking, it consists in inducing a suitable increase of the rate of the Poisson distribution of the number of bilateral trajectories with label at most $u$, which enter $\left(N K^{\delta}\right) \cap \mathbb{Z}^{d}$. The lower bound (0.7) ii) shows that this intuitive strategy is sub-optimal, see Remark 6.5 4). In essence, this strategy leads to a version of (0.7) ii) where $(\sqrt{a}-\sqrt{u})^{2}$ is replaced by the strictly bigger quantity $a \log \frac{a}{u}-a+u$.

Further, it is known that the vacant set $\mathcal{V}^{u}$ of random interlacements at level $u$ undergoes a phase transition between a percolative phase when $u<u_{*}$, and a non-percolative phase when $u>u_{*}$, for a certain critical level $u_{*} \in(0, \infty)$ (see [28], [25], and also [12], [22] for recent developments). When $u<u_{*}$, the vacant set of random interlacements is in a percolative regime. In the context of Bernoulli percolation, disconnecting a large macroscopic body in the percolative phase would involve an exponential cost proportional to $N^{d-1}$ (and surface tension), in the spirit of the study of the presence of a large finite cluster at the origin, see p. 216 of [15], and Theorem 2.5, p. 16 of [4]. In the present context, one may wonder whether large deviations of the density profile, as in (0.7), with an exponential cost proportional to $N^{d-2}$, capture the main mechanism ensuring that a macroscopic body gets disconnected from infinity by the interlacement at level $u$, when $u<u_{*}$. We refer to Remark 6.5 5) and to [18] for more on this topic.

It is also of interest to point out that the large deviation principle (0.2) has some similar flavor to results of [3] concerning the Gaussian free field (although the approaches in the two articles are quite different). This feature is in line with the isomorphism theorem of [30], which relates the field of occupation times of random interlacements to the square of Gaussian free fields.

Let us give some comments concerning proofs. The large deviation principle (0.6) (concerning the profile of the Brownian occupation-time measure) is used as a step in the proof of (0.2). Due to the scaling property of $\mathcal{L}_{\alpha}$, see (1.25), $\nu_{L, \alpha}$ has the same distribution as $\widetilde{\nu}_{L, \alpha}$, the restriction to $B$ of $\frac{1}{L^{d-2}} \mathcal{L}_{\alpha L^{d-2}}$. Since $\mathcal{L}_{\alpha}$ has stationary and independent increments, (0.6) can be proved by means of general Cramér theory and sub-additivity, see for instance [10], p. 252. In this process, one important ingredient is a new identity for the Laplace functional of $\mathcal{L}_{\alpha}$, which is based on methods of Schrödinger semi-groups, see [6], [7], [8]. Indeed, we show in Theorem 2.2 that for $\alpha \geq 0$, and any bounded measurable function $V$ on $\mathbb{R}^{d}$, with compact support,

$$
\begin{aligned}
& \mathbb{E}\left[e^{\left\langle\mathcal{L}_{\alpha}, V\right\rangle}\right]=e^{\alpha \Gamma(V)}, \text { where } \\
& \Gamma(V)=\int_{\mathbb{R}^{d}} V d y+\sup _{\varphi \in L^{2}\left(\mathbb{R}^{d}\right)}\left\{2 \int_{\mathbb{R}^{d}} V \varphi d y+\int_{\mathbb{R}^{d}} V \varphi^{2} d y-\mathcal{E}(\varphi, \varphi)\right\} \in(-\infty,+\infty],
\end{aligned}
$$

and for $\varphi \in L^{2}\left(\mathbb{R}^{d}\right)$,

$$
\mathcal{E}(\varphi, \varphi)=\left\{\begin{array}{l}
\frac{1}{2} \int_{\mathbb{R}^{d}}|\nabla \varphi|^{2} d y, \text { if } \varphi \in H^{1}\left(\mathbb{R}^{d}\right) \\
+\infty, \text { otherwise, }
\end{array}\right.
$$


stands for the Dirichlet form attached to the Brownian semi-group (acting on $L^{2}\left(\mathbb{R}^{d}\right)$ ), see also below (2.4). Both members of (0.8) may well be infinite. Remarkably, and unlike Proposition 2.6 of [31] (see also Remark 2.1 below), (0.8) is an identity between extended numbers in $(0,+\infty]$, which does not require any smallness assumption on $V$. We also refer to Remark 2.3 for the discrete space counterpart of this identity.

The proof of the main large deviation principle (0.2) on the density profile $\rho_{N, u}$ appears in Theorem 5.8. It relies on sub-additivity and naturally splits into a lower bound and an upper bound. On the one hand, the lower bound (proved in Theorem 5.4) first relies on a lower bound stemming from sub-additivity. It then exploits a combination of the fact that the Brownian occupation-time measure is a scaling limit of occupation times of random interlacements on $\mathbb{Z}^{d}$ (as proved in [31] and recalled in (4.5) below), and the key large deviation result on $\frac{1}{\alpha} \mathcal{L}_{\alpha}$ restricted to a box (proved in Section 3). On the other hand, the upper bound (proved in Theorem 5.5) combines an upper bound stemming from sub-additivity, involving a discrete version of the functional in (0.3), and an estimate in the spirit of Gamma-convergence (see Chapter 7 of [9]), which compares the large $N$ behavior of a sequence of variational problems on the scaled lattices $\frac{1}{N} \mathbb{Z}^{d}$ to a suitable continuous-space variational problem.

The asymptotic bounds (0.7) on the probability of insulation of a large macroscopic body by high values of the (smoothed-out) occupation-time profile (see Theorems 6.2 and 6.4 ) are direct applications of the main large deviation principle (0.2) and of the structure of the rate function, see Lemma 3.1.

Let us now describe the organization of this article. Section 1 introduces further notation, collects material concerning Schrödinger semi-groups, and recalls some properties of Brownian interlacements. The main objective of Section 2 is to establish the identity (2.5) in Theorem 2.2. Some of the key consequences of this identity appear in the Corollaries 2.4 and 2.5. The discrete space situation is discussed in Remark 2.3. Section 3 derives in Theorem 3.2 a large deviation principle for the Brownian interlacement case, which plays a central role. Its application to the proof of (0.6) appears in Corollary 3.3. The main properties of the rate function $I_{v}$ are collected in Lemma 3.1. The short Section 4 describes the (scaled) discrete space set-up and some of the results following by the methods of Section 2 in this context, see Theorem 4.1 and Corollary 4.2. Section 5 is devoted to the proof of (0.2), see Theorem 5.8. The lower bound appears in Theorem 5.4 and the upper bound in Theorem 5.5. The main sub-additive estimates are contained in Corollary 5.3, and the relevant form of the scaling limit of occupation times in Lemma 5.1. The last Section 6 contains the proof of (0.7), see Theorems 6.2 and 6.4. Extensions are discussed at the end, in Remark 6.5.

Finally, let us explain the convention we use concerning constants. We denote by $c, c^{\prime}, \bar{c}$ positive constants changing from place to place, which simply depend on $d$. Numbered constants $c_{0}, c_{1}, \ldots$ refer to the value corresponding to their first appearance in the text. Dependence of constants on additional parameters appears in the notation. 


\section{Some useful facts about Schrödinger semi-groups and Brownian interlacements}

In this section, we first introduce some further notation. We collect some rather classical properties of Schrödinger semi-groups and gauge functions, which will be useful in the next section, see Proposition 1.2 and Lemma 1.3. Moreover, we recall some properties of Brownian interlacements on $\mathbb{R}^{d}$. Further facts concerning continuous time random interlacements on $\mathbb{Z}^{d}$ will appear in Section 4 . Throughout, we tacitly assume that $d \geq 3$.

We write $|\cdot|$ and $|\cdot|_{\infty}$ for the Euclidean and the supremum norms on $\mathbb{R}^{d}$. We denote by $B(y, r)$ the closed Euclidean ball with center $y \in \mathbb{R}^{d}$ and radius $r \geq 0$. We write $B_{\infty}(y, r)$ in the case of the supremum norm. Given $A, B \subseteq \mathbb{R}^{d}$, we denote by $d(A, B)=\inf \left\{\left|y-y^{\prime}\right|\right.$; $\left.y \in A, y^{\prime} \in B\right\}$ the mutual Euclidean distance of $A$ an $B$. When $A=\{y\}$, we simply write $d(y, B)$ in place of $d(\{y\}, B)$. We define $d_{\infty}(A, B)$ and $d_{\infty}(y, B)$ analogously, with $|\cdot|_{\infty}$ in place of $|\cdot|$. The shorthand notation $K \subset \subset \mathbb{R}^{d}$, resp. $K \subset \subset \mathbb{Z}^{d}$, means that $K$ is compact subset of $\mathbb{R}^{d}$, resp. a finite subset of $\mathbb{Z}^{d}$. We denote by $f_{+}=\max \{f, 0\}$, $f_{-}=\max \{-f, 0\}$, the positive and negative part of a function $f$. We routinely write $\langle\nu, f\rangle$ to denote the integral with respect to a measure $\nu$ of a measurable, non-negative, or $\nu$-integrable, function $f$. When $f, h$ are measurable functions on $\mathbb{R}^{d}$ such that $|f h|$ is Lebesgue-integrable, we write $\langle f, h\rangle=\int_{\mathbb{R}^{d}} f(y) h(y) d y$. We denote by $\|f\|_{\infty}$ the supremum norm of the function $f$, and by $\|f\|_{p}$ its $L^{p}$-norm $(1 \leq p<\infty)$. We specify the relevant $L^{p}$-space in the notation when there might be some ambiguity, and write for instance $\|f\|_{L^{p}\left(\mathbb{R}^{d}, d y\right)}$ or $\|f\|_{L^{p}(B, \nu)}$.

We denote by $W_{+}$the subspace of $C\left(\mathbb{R}_{+}, \mathbb{R}^{d}\right)$ of continuous $\mathbb{R}^{d}$-valued trajectories tending to infinity at infinite times. We write $\left(X_{t}\right)_{t \geq 0}$ for the canonical process, and denote by $\left(\theta_{t}\right)_{t \geq 0}$ the canonical shift. We endow $W_{+}$with the $\sigma$-algebra $\mathcal{W}_{+}$generated by the canonical process. Given an open set $U$ of $R^{d}, w \in W_{+}$, we write $T_{U}(w)=$ $\inf \left\{s \geq 0 ; X_{s}(w) \notin U\right\}$, for the exit time of $U$. When $F$ is a closed subset of $\mathbb{R}^{d}$, we write $H_{F}(w)=\inf \left\{s \geq 0 ; X_{s}(w) \in F\right\}$, and $\widetilde{H}_{F}(w)=\inf \left\{s>0 ; X_{s}(w) \in F\right\}$, for the respective entrance, and hitting times of $F$. We assume $d \geq 3$, so that Brownian motion on $\mathbb{R}^{d}$ is transient, and we view $P_{y}$, the Wiener measure starting from $y \in \mathbb{R}^{d}$, as defined on $\left(W_{+}, \mathcal{W}_{+}\right)$. We denote by $E_{y}$ the corresponding expectation. When $\rho$ is a finite measure on $\mathbb{R}^{d}$, we write $P_{\rho}$ for the Wiener measure with "initial distribution" $\rho$

and $E_{\rho}$ for the corresponding expectation. We write $p\left(t, y, y^{\prime}\right)=(2 \pi t)^{-\frac{d}{2}} \exp \left\{-\frac{\left|y-y^{\prime}\right|^{2}}{2 t}\right\}$, with $t>0, y, y^{\prime} \in \mathbb{R}^{d}$, for the Brownian transition density.

We now recall some properties of the Schrödinger semi-groups we consider here. We denote by $L_{c}^{\infty}\left(\mathbb{R}^{d}\right)$ the space of bounded measurable functions $V$ on $\mathbb{R}^{d}$, which vanish outside a compact subset of $\mathbb{R}^{d}$. Given $V \in L_{c}^{\infty}\left(\mathbb{R}^{d}\right)$, we introduce the Schrödinger semi-group attached to $V$, namely the strongly continuous self-adjoint semi-group on $L^{2}\left(\mathbb{R}^{d}, d y\right)$, see Proposition 3.3, p. 16 of [26],

$$
\begin{aligned}
R_{t}^{V} f(y) & =E_{y}\left[f\left(X_{t}\right) e^{\int_{0}^{t} V\left(X_{s}\right) d s}\right], \text { for } f \in L^{2}\left(\mathbb{R}^{d}\right), t \geq 0, y \in \mathbb{R}^{d}, \\
& =\int_{\mathbb{R}^{d}} r_{V}\left(t, y, y^{\prime}\right) f\left(y^{\prime}\right) d y^{\prime}, \text { when } t>0, \text { where } \\
r_{V}\left(t, y, y^{\prime}\right) & =p\left(t, y, y^{\prime}\right) E_{y, y^{\prime}}^{t}\left[\exp \left\{\int_{0}^{t} V\left(X_{s}\right) d s\right\}\right], \text { for } t>0, y, y^{\prime} \in \mathbb{R}^{d},
\end{aligned}
$$


is a symmetric function of $y, y^{\prime}$, see Proposition 3.1, p. 13-14 of [26], which is jointly continuous, see Proposition 3.5, p. 18 of [26], and $E_{y, y^{\prime}}^{t}$ stands for the expectation corresponding to $P_{y, y^{\prime}}^{t}$, the Brownian bridge measure in time $t$ from $y$ to $y^{\prime}$, see p. 137-140 of [26]. As an immediate consequence of (1.2),

$$
e^{-\|V\|_{\infty} t} p\left(t, y, y^{\prime}\right) \leq r_{V}\left(t, y, y^{\prime}\right) \leq e^{\|V\|_{\infty} t} p\left(t, y, y^{\prime}\right) \text {, for } t>0, y, y^{\prime} \in \mathbb{R}^{d} .
$$

We now turn to the discussion of the Green operators corresponding to the Schrödinger semi-groups. We thus consider $V \in L_{c}^{\infty}\left(\mathbb{R}^{d}\right)$ as above and define

$$
G_{V} f(y)=E_{y}\left[\int_{0}^{\infty} f\left(X_{s}\right) e^{\int_{0}^{s} V\left(X_{u}\right) d u} d s\right]=\int_{\mathbb{R}^{d}} \int_{0}^{\infty} r_{V}\left(s, y, y^{\prime}\right) f\left(y^{\prime}\right) d s d y^{\prime},
$$

for $f$ a measurable non-negative function on $\mathbb{R}^{d}$, and $y \in \mathbb{R}^{d}$.

When $V=0$ (so $\left.r_{V}\left(t, y, y^{\prime}\right)=p\left(t, y, y^{\prime}\right)\right)$ we simply write $G$, and recover the usual Green operator attached to Brownian motion

$$
G f(y)=E_{y}\left[\int_{0}^{\infty} f\left(X_{s}\right) d s\right]=\frac{\Gamma\left(\frac{d}{2}-1\right)}{2 \pi^{\frac{d}{2}}} \int_{\mathbb{R}^{d}} \frac{1}{\left|y-y^{\prime}\right|^{d-2}} f\left(y^{\prime}\right) d y .
$$

We introduce in the (classical) lemma below a condition corresponding to the so-called sub-criticality (of $\frac{1}{2} \Delta+V$ ) see p. 145, as well as pp. 129, 136, 150 of [21]. Our assumptions are slightly different and we briefly sketch the proof for the reader's convenience.

Lemma 1.1. (recall $V \in L_{c}^{\infty}\left(\mathbb{R}^{d}\right)$ )

Assume that

$$
\begin{aligned}
& G_{V} W\left(y_{0}\right)<\infty, \text { for some } y_{0} \in \mathbb{R}^{d} \text { and some }[0,1] \text {-valued, measurable } \\
& \text { function } W \text { not a.e. equal to } 0 .
\end{aligned}
$$

Then, for any bounded open set $U$,

$$
G_{V} 1_{U} \text { is a bounded function. }
$$

Moreover, in the notation of (1.1), the Schrödinger semi-group

$$
\left(R_{t}^{V}\right)_{t \geq 0} \text { is a strongly continuous semi-group of self-adjoint contractions }
$$
on $L^{2}\left(\mathbb{R}^{d}\right)$.

Proof. We first sketch the proof of (1.7). We note that

$$
r_{V}\left(1, y_{0}, y\right) \geq c\left(V, y_{0}, y_{1}\right) r_{V}\left(\frac{1}{2}, y_{1}, y\right) \text {, for } y_{0}, y_{1}, y \in \mathbb{R}^{d},
$$

with $c(V, \cdot, \cdot)$ locally bounded away from zero. Indeed, this follows from (1.3) and the inequality $p(1,0, z) / p\left(\frac{1}{2}, z_{1}, z\right)=2^{-\frac{d}{2}} \exp \left\{\frac{1}{2}\left|z-2 z_{1}\right|^{2}-\left|z_{1}\right|^{2}\right\} \geq 2^{-\frac{d}{2}} \exp \left\{-\left|z_{1}\right|^{2}\right\}$, for $z, z_{1} \in$ $\mathbb{R}^{d}$, combined with translation invariance (set $z=y-y_{0}, z_{1}=y_{1}-y_{0}$ ). Thus, for $y_{1} \in \mathbb{R}^{d}$, we find that the above inequality together with the semi-group property yields that

$$
\begin{aligned}
& \infty>G_{V} W\left(y_{0}\right) \stackrel{(1.4)}{\geq} \int_{0}^{\infty} \int r_{V}\left(s+1, y_{0}, y\right) W(y) d y d s \geq \\
& c\left(V, y_{0}, y_{1}\right) \int_{\frac{1}{2}}^{\infty} \int r_{V}\left(t, y_{1}, y\right) W(y) d y d t,
\end{aligned}
$$


which combined with (1.3) (for the values $\left.t \in\left(0, \frac{1}{2}\right]\right)$ implies that $G_{V} W$ is locally bounded. In addition, by the semi-group property, we see that for $y \in \mathbb{R}^{d}$,

$$
G_{V} W(y) \geq \int_{0}^{\infty} \int r_{V}\left(s+1, y, y^{\prime}\right) W\left(y^{\prime}\right) d y^{\prime} d s \geq G_{V} 1_{U}(y) \inf _{z \in U} R_{1}^{V} W .
$$

By our assumption on $W$, the last term is positive, and hence, $G_{V} 1_{U}$ is locally bounded. Choosing $K \subset \subset \mathbb{R}^{d}$ containing $U$ and the support of $V$, the strong Markov property yields,

$$
G_{V} 1_{U}(y)=E_{y}\left[\left(G_{V} 1_{U}\right)\left(X_{H_{K}}\right), H_{K}<\infty\right] \text {, for any } y \in \mathbb{R}^{d},
$$

so $G_{V} 1_{U}$ is bounded and (1.7) follows.

We now turn to the proof of (1.8). For $\varphi \in L_{c}^{\infty}\left(\mathbb{R}^{d}\right)$ we denote by $d E_{\varphi, \varphi}(\lambda)$ the spectral measure of $\varphi$ (and $E$ is a spectral resolution of the identity of the generator of $\left(R_{t}^{V}\right)_{t \geq 0}$, see for instance Theorems 13.30 and 13.37, pp. 348, 360 of [24]). We find by (1.7) that

$$
\infty>\int_{0}^{\infty} d t\left\langle R_{t}^{V} \varphi, \varphi\right\rangle=\int_{0}^{\infty} d t \int_{\mathbb{R}} e^{-\lambda t} d E_{\varphi, \varphi}(\lambda),
$$

Hence, $d E_{\varphi, \varphi}$ gives no mass to $(-\infty, 0]$, and therefore for $\varphi \in L_{c}^{\infty}\left(\mathbb{R}^{d}\right)$,

$$
\left\langle R_{t}^{V} \varphi, R_{t}^{V} \varphi\right\rangle=\int_{0}^{\infty} e^{-2 \lambda t} d E_{\varphi, \varphi}(\lambda) \leq \int_{0}^{\infty} d E_{\varphi, \varphi}(\lambda)=\|\varphi\|_{L^{2}\left(\mathbb{R}^{d}\right)}^{2} .
$$

Since $L_{c}^{\infty}\left(\mathbb{R}^{d}\right)$ is dense in $L^{2}\left(\mathbb{R}^{d}\right)$, the claim (1.8) follows.

We now recall some properties of the gauge function

$$
\gamma_{V}(y)=E_{y}\left[e^{\int_{0}^{\infty} V\left(X_{s}\right) d s}\right], \text { for } y \in \mathbb{R}^{d}
$$

(note that $V \in L_{c}^{\infty}\left(\mathbb{R}^{d}\right)$ and the integral is finite due to transience, or more precisely, to the fact that $P_{y}$ is a probability on $W_{+}$, see above (1.1)). As the next proposition shows, the gauge function is closely related to the Schrödinger semi-group (attached to $V$ ) via its Green operator. We refer to [6], [7], and [8], for much more on the subject.

Proposition 1.2. (recall $V \in L_{c}^{\infty}\left(\mathbb{R}^{d}\right)$ )

The condition (1.6) is equivalent to $\gamma_{V}$ is not identically infinite (Gauge Condition).

If (1.6), or equivalently (1.10), holds, then

$$
\gamma_{V} \text { is a bounded continuous function on } \mathbb{R}^{d} \text { tending to } 1 \text { at infinity, }
$$

$$
\gamma_{V}=1+G V \gamma_{V}=1+G_{V} V \text {. }
$$

Proof. To show that (1.6) implies (1.10), we use the identity

$$
e^{\int_{0}^{t} V\left(X_{s}\right) d s}=1+\int_{0}^{t} V\left(X_{s}\right) e^{\int_{0}^{s} V\left(X_{u}\right) d u} d s, \text { for } t \geq 0 .
$$


By Fatou's lemma, (1.13) implies that $\gamma_{V}(y) \leq 1+G_{V}|V|(y)$, which is a bounded function of $y$, by (1.7). The fact that $\gamma_{V}$ coincides with the last expression of (1.12) follows by dominated convergence.

To prove that (1.10) implies (1.6), either $V_{+}(=\max (V, 0))$ vanishes a.e., so that $r_{V} \leq p$ and (1.6) holds, or else, by Theorem 2.8, p. 4651 of [6], $\left\|G_{V} V_{+}\right\|_{\infty}<\infty$ and $V_{+}$is not a.e. equal to 0 . This implies (1.6) (choosing $W=V_{+} \wedge 1$ ). Thus (1.6) and (1.10) are equivalent.

To prove (1.11), we already know from the discussion below (1.13) that $\gamma_{V}$ is a bounded function. It is continuous by the Corollary, p. 150 of [8], or (ii) in Theorem 4.7, p. 115 of the same reference. The fact that $\gamma_{V}$ tends to 1 at infinity follows from the first equality of (1.12), which we prove now. To derive the first equality of (1.12) (and this will compete the proof of Proposition 1.2), we use the identity

$$
e^{\int_{0}^{t} V\left(X_{s}\right) d s}=1+\int_{0}^{t} V\left(X_{s}\right) e^{\int_{s}^{t} V\left(X_{u}\right) d u} d s, \text { for } t \geq 0 .
$$

Integrating with respect to $P_{y}$ and using the Markov property yields

$$
E_{y}\left[e^{\int_{0}^{t} V\left(X_{s}\right) d s}\right]=1+E_{y}\left[\int_{0}^{t} V\left(X_{s}\right) E_{X_{s}}\left[e^{\int_{0}^{t-s} V\left(X_{u}\right) d u}\right] d s\right] \text {, for } y \in \mathbb{R}^{d} .
$$

By (1.7) and (1.13) we see that the inner expectation is uniformly bounded, and converges to $\gamma_{V}\left(X_{s}\right)$ as $t \rightarrow \infty$. The first equality of (1.12) now follows by dominated convergence. The second equality results from the discussion below (1.13), and the proof of Proposition 1.2 is now complete.

The following approximation lemma will be helpful in Sections 2 and 3. We say that $r$ and $r^{\prime}$ in $(1, \infty)$ are conjugate exponents when $\frac{1}{r}+\frac{1}{r^{\prime}}=1$.

Lemma 1.3. Consider $r>\frac{d}{2}$, and $V, V_{n}, n \geq 1$, in $L_{c}^{\infty}\left(\mathbb{R}^{d}\right)$, which all vanish outside $K \subset \subset \mathbb{R}^{d}$. Assume that (1.6) holds for $V$ and that

$$
\lim _{n}\left\|V-V_{n}\right\|_{L^{r}\left(\mathbb{R}^{d}\right)}=0 .
$$

Then, for large $n$, (1.6) holds for $V_{n}$, and

$$
\gamma_{V_{n}} \text { converges uniformly to } \gamma_{V} \text { on } \mathbb{R}^{d} \text {. }
$$

Proof. By Theorem 2.17, p. 4660 of [6] (Super Gauge Theorem), for some $p>1, p V$ satisfies (1.10) and hence (1.6). Thus, denoting by $q$ the conjugate exponent of $p$,

$$
\begin{aligned}
\left|\gamma_{V_{n}}(y)-\gamma_{V}(y)\right| & \leq E_{y}\left[\left|e^{\int_{0}^{\infty}\left(V_{n}-V\right)\left(X_{s}\right) d s}-1\right| e^{\int_{0}^{\infty} V\left(X_{s}\right) d s}\right] \\
& \stackrel{\text { Hölder }}{\leq} E_{y}\left[\left|e^{\int_{0}^{\infty}\left(V_{n}-V\right)\left(X_{s}\right) d s}-1\right|^{q}\right]^{\frac{1}{q}}\left\|\gamma_{p V}\right\|_{\infty}^{\frac{1}{p}}, \text { for all } y \in \mathbb{R}^{d}
\end{aligned}
$$

(and $\left\|\gamma_{p V}\right\|_{\infty}$ is finite by $(1.11)$ ). 
Thus, choosing $2 m \geq q$, with $m \geq 1$ integer, and setting $\Delta_{n}=V_{n}-V$, we find that the $(2 m)$-th power of the first term in the last line of (1.17) is smaller than

$$
E_{y}\left[\left(e^{\int_{0}^{\infty} \Delta_{n}\left(X_{s}\right) d s}-1\right)^{2 m}\right]=\sum_{\ell=0}^{2 m}\left(\begin{array}{c}
2 m \\
\ell
\end{array}\right)(-1)^{2 m-\ell} E_{y}\left[e^{\int_{0}^{\infty} \ell \Delta_{n}\left(X_{s}\right) d s}\right] .
$$

The claim (1.16) will thus follow once we show that for each fixed $\ell \geq 0$,

$$
\sup _{y \in \mathbb{R}^{d}}\left|E_{y}\left[e^{\ell \int_{0}^{\infty} \Delta_{n}\left(X_{s}\right) d s}\right]-1\right| \underset{n}{\longrightarrow} 0 .
$$

Expanding the exponential and using the Markov-property, we can bound the absolute value in (1.18) by $\sum_{k \geq 1} \ell^{k}\left\|G\left|\Delta_{n}\right|\right\|_{\infty}^{k}$.

By assumption, $r>\frac{d}{2}$, so, the conjugate exponent $r^{\prime}$ belongs to $\left(1, \frac{d}{d-2}\right)$. Then, for $z \in \mathbb{R}^{d}$

$$
\begin{aligned}
G\left|\Delta_{n}\right|(z) \stackrel{(1.5)}{=} c \int \frac{\left|\Delta_{n}(y)\right|}{|y-z|^{d-2}} d y \stackrel{\text { Hölder }}{\leq} c\left(\int_{K} \frac{1}{|y-z|^{(d-2) r^{\prime}}} d y\right)^{\frac{1}{r^{\prime}}}\left\|\Delta_{n}\right\|_{r} \\
\leq c(r, K)\left\|\Delta_{n}\right\|_{r}
\end{aligned}
$$

(for the last bound, where the dependence in $z$ has disappeared, one considers the smallest $R \geq 1$ such that $B(0, R) \supseteq K$, and looks separately at $z \notin B(0,2 R)$, or $z \in B(0,2 R)$ and hence $K \subseteq B(z, 3 R)$ ). So, we see that for $\ell \leq 2 m$,

$$
\begin{aligned}
\sup _{y \in \mathbb{R}^{d}}\left|E_{y}\left[e^{\ell \int_{0}^{\infty} \Delta_{n}\left(X_{s}\right) d s}\right]-1\right| & \leq \sum_{k \geq 1}(2 m)^{k}\left\|G\left|\Delta_{n}\right|\right\|_{\infty}^{k} \\
& \leq \frac{c(r, K) 2 m\left\|\Delta_{n}\right\|_{r}}{\left(1-c(r, K) 2 m\left\|\Delta_{n}\right\|_{r}\right)_{+}} \underset{n}{\stackrel{(1.5)}{\longrightarrow}} 0 .
\end{aligned}
$$

This proves (1.18) and (1.16) follows.

We now recall some properties of Brownian interlacements, and refer to Section 2 of [31] for more detail. Brownian interlacements correspond to a certain Poisson point process on a state space, which is the product $W^{*} \times \mathbb{R}_{+}$, where $W^{*}$ stands for the space of continuous trajectories from $\mathbb{R}$ into $\mathbb{R}^{d}$, tending to infinity at plus and minus infinite times, modulo time-shift. The intensity measure of this Poisson point process is the product of a certain $\sigma$-finite measure $\nu$ on $W^{*}$ (see Theorem 2.2 of [31]), with the Lebesgue measure $d \alpha$ on $\mathbb{R}_{+}$. Informally, this point process corresponds to a cloud of doubly-infinite trajectories modulo time-shift having each a non-negative label (the $\mathbb{R}_{+}$-component of $W^{*} \times \mathbb{R}_{+}$). The Poisson point process is defined on a certain canonical space $(\Omega, \mathcal{A}, \mathbb{P})$, see $(2.23)$ of [31]. We collect below some properties of Brownian interlacements, which we will use here.

Given $K \subset \subset \mathbb{R}^{d}, \alpha \geq 0$, and $\omega \in \Omega$, one considers the point measure on $W_{+}$, denoted by $\mu_{K, \alpha}(\omega)$, which collects for all bilateral trajectories with label at most $\alpha$, which enter $K$ at some point, their forward trajectories after their first entrance time in $K$. Then, see (2.25) of [31],

$$
\mu_{K, \alpha} \text { is a Poisson point process on } W_{+} \text {with intensity measure } \alpha P_{e_{K}} \text {, where }
$$
$e_{K}(d y)$ stands for the equilibrium measure of $K$. 
The equilibrium measure of $K$ is a finite measure concentrated on $\partial K$, and its total mass is called the capacity of $K$, see pp. 58, 61 of [26]. Moreover,

$$
\left\langle e_{K}, G f\right\rangle=\langle 1, f\rangle \text { if } f \in L_{c}^{\infty}\left(\mathbb{R}^{d}\right) \text { vanishes outside } K .
$$

One also introduces the occupation-time measure $\mathcal{L}_{\alpha}(\omega)$ of Brownian interlacements at level $\alpha \geq 0$ in the cloud $\omega$. It is the Radon measure on $\mathbb{R}^{d}$, which to each $A \in \mathcal{B}\left(\mathbb{R}^{d}\right)$ gives a mass equal to the total time spent in $A$ by all trajectories modulo time-shift with label at most $\alpha$ in the cloud $\omega$. In particular, when $V \in L_{c}^{\infty}\left(\mathbb{R}^{d}\right)$ vanishes outside $K \subset \subset \mathbb{R}^{d}$, one has for $\alpha \geq 0$, and $\omega \in \Omega$,

$$
\left\langle\mathcal{L}_{\alpha}(\omega), V\right\rangle=\left\langle\mu_{K, \alpha}(\omega), f_{V}\right\rangle \text {, where } f_{V}(w)=\int_{0}^{\infty} V\left(X_{s}(w)\right) d s, \text { for } w \in W_{+} .
$$

The intensity measure of $\mathcal{L}_{\alpha}$ equals $\alpha d y$, cf. (2.38) of [31], that is

$$
\mathbb{E}\left[\left\langle\mathcal{L}_{\alpha}, V\right\rangle\right]=\alpha \int_{\mathbb{R}^{d}} V d y, \text { for all } V \in L_{c}^{\infty}\left(\mathbb{R}^{d}\right) .
$$

Moreover, $\mathcal{L}_{\alpha}$ has an important scaling property, see (2.43) of [31],

$$
\mathcal{L}_{\lambda^{2-d} \alpha} \stackrel{\text { law }}{=} \lambda^{2} h_{\lambda} \circ \mathcal{L}_{\alpha}, \text { for } \lambda>0,
$$

where $h_{\lambda} \circ \mathcal{L}_{\alpha}$ stands for the image of $\mathcal{L}_{\alpha}$ under the homothety of ratio $\lambda$ on $\mathbb{R}^{d}$.

One also has an expression for the Laplace transform of $\mathcal{L}_{\alpha}$ in the "neighborhood of the origin" (see Proposition 2.6 of [31]). Namely for $V \in L_{c}^{\infty}\left(\mathbb{R}^{d}\right)$ such that $\|G|V|\|_{\infty}<1$, one has

$$
\mathbb{E}\left[e^{\left\langle\mathcal{L}_{\alpha}, V\right\rangle}\right]=\exp \left\{\alpha\left\langle V,(I-G V)^{-1} 1\right\rangle\right\}
$$

(the assumption on $V$ ensures that $I-G V$ operating on $L^{\infty}\left(\mathbb{R}^{d}\right)$ has a bounded inverse). In the next section we will derive identities that bypass the smallness assumption on $V$ in (1.26), and remain true even when the left-hand side of (1.26) explodes.

We close this section with a lemma about Poisson point processes, which will be helpful in the next section. We consider a measurable space $(E, \mathcal{E})$.

Lemma 1.4. Let $\mu$ be a Poisson point process on $E$ with finite intensity measure $\eta$ (i.e. $\eta(E)<\infty)$, and let $\Phi: E \rightarrow \mathbb{R}$ be a measurable function. Then, one has

$$
\mathbb{E}\left[e^{\langle\mu, \Phi\rangle}\right]=\exp \left\{\int_{E}\left(e^{\Phi}-1\right) d \eta\right\}
$$

(this is an identity between numbers in $(0,+\infty])$.

Proof. Set $\Phi_{n}=\sum_{\mathbb{Z} \ni k<n 2^{n}} \frac{k}{2^{n}} 1\left\{\frac{k}{2^{n}} \leq \Phi<\frac{k+1}{2^{n}}\right\}$, for $n \geq 1$, so that $\Phi_{n}$ is measurable, $(-\infty, n]$-valued, and $\Phi_{n}$ increases to $\Phi$, as $n \rightarrow \infty$. Then, for each $n$, classically, see for instance [23], p. 130-134,

$$
\mathbb{E}\left[e^{\left\langle\mu, \Phi_{n}\right\rangle}\right]=\exp \left\{\int_{E}\left(e^{\Phi_{n}}-1\right) d \eta\right\}\left(<\infty, \text { since } \Phi_{n} \leq n \text { and } \eta(E)<\infty\right),
$$

and (1.27) follows by monotone convergence. 


\section{Laplace functional of occupation-time measures of Brownian interlacements}

In this section, with the help of Schrödinger semi-groups techniques (in particular Lemma 1.1 and Proposition 1.2 of the previous section), we derive an identity for the Laplace functional of $\mathcal{L}_{\alpha}$, see Theorem 2.2. This identity plays an important role for the identification of the rate function governing the large deviation principle for the occupation-time profile of Brownian interlacements in a large box, which we derive in the next section. We state two consequences of the basic identity (2.5), see Corollaries 2.4 and 2.5. In Remark 2.3, we discuss the corresponding identity one obtains in the case of continuous-time random interlacements on a transient weighted graph.

We begin with the observation that the Laplace functional of $\mathcal{L}_{\alpha}$ naturally involves the gauge function. We consider $V \in L_{c}^{\infty}\left(\mathbb{R}^{d}\right)$ and recall that $\gamma_{V}$ stands for the gauge function, see (1.9). When $V$ vanishes on $K^{c}$, with $K \subset \subset \mathbb{R}^{d}$, as in (1.23), we can express $\left\langle\mathcal{L}_{\alpha}, V\right\rangle$ in terms of the Poisson point process $\mu_{K, \alpha}$ introduced above (1.20), and use Lemma 1.4 to compute the exponential moment of $\left\langle\mathcal{L}_{\alpha}, V\right\rangle$. We find

$$
\begin{gathered}
\mathbb{E}\left[e^{\left\langle\mathcal{L}_{\alpha}, V\right\rangle}\right] \stackrel{(1.23)}{=} \mathbb{E}\left[e^{\left\langle\mu_{K, \alpha}, f_{V}\right\rangle}\right] \stackrel{(1.20),(1.27)}{=} e^{\alpha\left\langle e_{K}, E_{x}\left[e^{\int_{0}^{\infty} V\left(X_{s}\right) d s}\right]-1\right\rangle} \\
\stackrel{(1.9)}{=} e^{\alpha\left\langle e_{K}, \gamma_{V}-1\right\rangle}, \text { for } \alpha \geq 0
\end{gathered}
$$

(note that this is an identity between numbers in $(0, \infty]$, and there is no smallness assumption on $V$ ).

Remark 2.1. It is well-known that the gauge function $\gamma_{V}$ (and hence the left-hand side of (2.1)) may very well be infinite, see for instance [21], p. 227, and pp. 166, 167. This feature complicates the study of the Laplace transform of $\mathcal{L}_{\alpha}$. When (1.6) (or equivalently (1.10)) holds, we know from (1.11), (1.12) that $\gamma_{V}$ is bounded continuous and $\gamma_{V}-1=G V \gamma_{V}$. By (1.22) we thus find that

$$
\mathbb{E}\left[e^{\left\langle\mathcal{L}_{\alpha}, V\right\rangle}\right]=e^{\alpha\left\langle V, \gamma_{V}\right\rangle}, \text { for } \alpha \geq 0, \text { when (1.6) holds. }
$$

In particular, when $\|G|V|\|_{\infty}<1$, then (1.10) holds (see for instance (2.6) in [29]), and $I-G V$ operating on $L^{\infty}\left(\mathbb{R}^{d}\right)$ has a bounded inverse. So, by the first equality in (1.12), $\gamma_{V}=(I-G V)^{-1} 1$, and we find $\left\langle V, \gamma_{V}\right\rangle=\left\langle V,(I-G V)^{-1} 1\right\rangle$. In this fashion we recover (1.26) out of $(2.2)$ when $\|G|V|\|_{\infty}<1$.

We introduce a notation for the logarithm of the Laplace functional of $\mathcal{L}_{1}$. For $V \in$ $L_{c}^{\infty}\left(\mathbb{R}^{d}\right)$, we set

$$
\Lambda(V)=\frac{1}{\alpha} \log \mathbb{E}\left[e^{\left\langle\mathcal{L}_{\alpha}, V\right\rangle}\right] \in(-\infty,+\infty](\text { and } \alpha>0 \text { is arbitrary by }(2.1)) .
$$

We also recall from (0.9) the notation

$$
\Gamma(V)=\int_{\mathbb{R}^{d}} V d y+\sup _{\varphi \in L^{2}\left(\mathbb{R}^{d}\right)}\{2\langle V, \varphi\rangle+\langle V \varphi, \varphi\rangle-\mathcal{E}(\varphi, \varphi)\},
$$


where $\mathcal{E}(\varphi, \varphi)$ denotes as in (0.10) the Dirichlet form attached to the Brownian semigroup acting on $L^{2}\left(\mathbb{R}^{d}\right)$ (i.e. $\left(R_{t}^{V=0}\right)_{t \geq 0}$ in the notation of (1.11)), see for instance [26], p. 26. Further, we will also use the notation $\mathcal{E}(\varphi, \varphi)$, when $\varphi$ belongs to the extended Dirichlet space $\mathcal{F}_{e}$ (consisting of functions that are a.e. limits of sequences in $H^{1}\left(\mathbb{R}^{d}\right)$ that are Cauchy for $\mathcal{E}(\cdot, \cdot))$, see Chapter $1 \S 5$ of [13].

We are now ready for the key identity of this section. The arguments we use are general and easily adapted to the context of continuous-time random interlacements on transient weighted graphs, as explained in Remark 2.3 below.

\section{Theorem 2.2.}

$$
\Lambda(V)=\Gamma(V), \text { for all } V \in L_{c}^{\infty}\left(\mathbb{R}^{d}\right) .
$$

Proof. We first assume $\Lambda(V)<\infty$ and show the identity (2.5). By (2.1), we know that (1.10), and hence (1.6) hold. By (2.2) we have

$$
\Lambda(V)=\left\langle V, \gamma_{V}\right\rangle \stackrel{(1.12)}{=}\langle V, 1\rangle+\left\langle V, G_{V} V\right\rangle .
$$

Similarly, we have

$$
\begin{aligned}
\Gamma(V) & =\langle V, 1\rangle+\Psi(V), \text { where } \\
\Psi(V) & =\sup _{\varphi \in L^{2}\left(\mathbb{R}^{d}\right)}\{2\langle V, \varphi\rangle+\langle V \varphi, \varphi\rangle-\mathcal{E}(\varphi, \varphi)\} \\
& =\sup _{\varphi \in L^{2}\left(\mathbb{R}^{d}\right)} \sup _{\varepsilon>0}\{2\langle V, \varphi\rangle+\langle(V-\varepsilon) \varphi, \varphi\rangle-\mathcal{E}(\varphi, \varphi)\} \\
& =\sup _{\varepsilon>0} \sup _{\varphi \in L^{2}\left(\mathbb{R}^{d}\right)}\{2\langle V, \varphi\rangle+\langle(V-\varepsilon) \varphi, \varphi\rangle-\mathcal{E}(\varphi, \varphi)\} .
\end{aligned}
$$

We know by (1.8) that the Schrödinger semi-group $\left(R_{t}^{V}\right)_{t \geq 0}$ is a semi-group of self-adjoint contractions on $L^{2}\left(\mathbb{R}^{d}\right)$. Its quadratic form is $\mathcal{E}(\varphi, \varphi)-\langle V \varphi, \varphi\rangle$, see for instance [6], p. 4654. Then, by Lemma 4.4, p. 22 of [26], and also below (4.10), p. 23 of the same reference, we have

$$
\sup _{\varphi \in L^{2}\left(\mathbb{R}^{d}\right)}\{2\langle V, \varphi\rangle+\langle(V-\varepsilon) \varphi, \varphi\rangle-\mathcal{E}(\varphi, \varphi)\}=\left\langle V, G_{V-\varepsilon} V\right\rangle,
$$

where $G_{V-\varepsilon}$ is defined as in (1.4) with $V$ replaced by $V-\varepsilon$. Note that the left-hand side of (2.8) is a decreasing function of $\varepsilon$, so the same holds for the right-hand side. As a result, we find that

$$
\Psi(V)=\sup _{\varepsilon>0}\left\langle V, G_{V-\varepsilon} V\right\rangle=\lim _{\varepsilon \rightarrow 0}\left\langle V, G_{V-\varepsilon} V\right\rangle=\left\langle V, G_{V} V\right\rangle,
$$

using dominated convergence and (1.7) in the last step. We have thus shown that

$$
\Gamma(V) \stackrel{(2.7)}{=}\langle V, 1\rangle+\Psi(V) \stackrel{(2.9)}{=}\langle V, 1\rangle+\left\langle V, G_{V} V\right\rangle \stackrel{(2.6)}{=} \Lambda(V),
$$

that is, (2.5) holds when $\Lambda(V)<\infty$. 
We now assume that $\Gamma(V)<\infty$, and show (2.5). Since $\Gamma(V)<\infty$, we have

$$
\sup _{\varphi \in L^{2}\left(\mathbb{R}^{d}\right)}\langle V \varphi, \varphi\rangle-\mathcal{E}(\varphi, \varphi) \leq 0
$$

(otherwise the supremum in (2.4) would be infinite). Since $\mathcal{E}(\varphi, \varphi)-\langle V \varphi, \varphi\rangle$ is the quadratic form associated to the strongly continuous self-adjoint Schrödinger semi-group $\left(R_{t}^{V}\right)_{t \geq 0}$ on $L^{2}\left(\mathbb{R}^{d}\right)$, it follows that $R_{t}^{V}, t \geq 0$, are contractions.

We first discuss the case where $V_{-}=0$ a.e.. If $V=0$ a.e., then (2.5) is immediate. Otherwise, $V_{+}$is not a.e. equal to 0 . We then apply the same considerations as below (2.7) to find that for $\varepsilon>0, \sup _{\varphi \in L^{2}\left(\mathbb{R}^{d}\right)}\{2\langle V, \varphi\rangle+\langle(V-\varepsilon) \varphi, \varphi\rangle-\mathcal{E}(\varphi, \varphi)\}=\left\langle V, G_{V-\varepsilon} V\right\rangle$, and that this quantity increases to $\sup _{\varphi \in L^{2}\left(\mathbb{R}^{d}\right)}\{2\langle V, \varphi\rangle+\langle V \varphi, \varphi\rangle-\mathcal{E}(\varphi, \varphi)\}$, as $\varepsilon$ tends to 0 . Coming back to the definition of $\Gamma(V)$ in $(2.4)$, we find that

$$
\infty>\Gamma(V)=\langle V, 1\rangle+\lim _{\varepsilon \rightarrow 0}\left\langle V, G_{V-\varepsilon} V\right\rangle=\langle V, 1\rangle+\left\langle V, G_{V} V\right\rangle,
$$

where we used monotone convergence in the last step. Hence, (1.6) holds and $\Lambda(V)<\infty$, by (2.2) and (1.11). The identity (2.5) follows from the first part of the proof.

If instead $V_{-}$is not a.e. equal to 0 , we define for $\lambda \in[0,1]$,

$$
V_{\lambda}=(1-\lambda) V-\lambda V_{-}=V-\lambda V_{+}\left(\in L_{c}^{\infty}\left(\mathbb{R}^{d}\right)\right),
$$

so that $V_{\lambda}$ increases to $V$ as $\lambda$ decreases to 0 . Note that

$$
\begin{aligned}
& 2\langle V, \varphi\rangle+\langle V \varphi, \varphi\rangle-\mathcal{E}(\varphi, \varphi) \geq 2\left\langle V_{\lambda}, \varphi\right\rangle+\left\langle V_{\lambda} \varphi, \varphi\right\rangle-\mathcal{E}(\varphi, \varphi)+A_{\lambda}, \text { where } \\
& A_{\lambda}=\inf _{\varphi} \lambda\left\{2\left\langle V_{+}, \varphi\right\rangle+\left\langle V_{+} \varphi, \varphi\right\rangle\right\} \stackrel{\text { Cauchy-Schwarz }}{\geq} \lambda \inf _{u \geq 0}\left\{-2\left\langle V_{+}, 1\right\rangle^{\frac{1}{2}} u+u^{2}\right\}>-\infty
\end{aligned}
$$

(in the second line, we used the Cauchy-Schwarz Inequality in $L^{2}\left(V_{+} d y\right)$ to write that $\left\langle V_{+}, \varphi\right\rangle \geq-\left\langle V_{+}, 1\right\rangle^{\frac{1}{2}}\left\langle V_{+} \varphi, \varphi\right\rangle^{\frac{1}{2}}$, and took a lower bound over $\left.u=\left\langle V_{+} \varphi, \varphi\right\rangle^{\frac{1}{2}}\right)$.

Since $\Gamma(V)<\infty$ by assumption, we see that

$$
\Gamma\left(V_{\lambda}\right)<\infty, \text { for } \lambda \in[0,1] .
$$

Moreover, for $0<\lambda \leq 1$, we have

$$
\begin{aligned}
& 2\langle V, \varphi\rangle+\langle V \varphi, \varphi\rangle-\mathcal{E}(\varphi, \varphi) \geq-2\left\langle V_{-}, \varphi\right\rangle+\left\langle V_{\lambda} \varphi, \varphi\right\rangle-\mathcal{E}(\varphi, \varphi)+B_{\lambda}, \text { where } \\
& B_{\lambda}=\inf _{\varphi} 2\left\langle V_{+}, \varphi\right\rangle+\lambda\left\langle V_{+} \varphi, \varphi\right\rangle>-\infty, \text { by a similar argument as below (2.13). }
\end{aligned}
$$

This shows that

$$
\infty>\sup _{\varphi \in L^{2}\left(\mathbb{R}^{d}\right)}\left\{-2\left\langle V_{-}, \varphi\right\rangle+\left\langle V_{\lambda} \varphi, \varphi\right\rangle-\mathcal{E}(\varphi, \varphi)\right\}
$$

In addition, by (2.14) and the argument below (2.10), the Schrödinger semi-group $\left(R_{t}^{V_{\lambda}}\right)_{t \geq 0}$ is a strongly continuous semi-group of self-adjoint contractions on $L^{2}\left(\mathbb{R}^{d}\right)$. From the argument below (2.7), we see that the above supremum in (2.16) equals $\lim _{\varepsilon \rightarrow 0}\left\langle V_{-}, G_{V_{\lambda}-\varepsilon} V_{-}\right\rangle=$ 
$\left\langle V_{-}, G_{V_{\lambda}} V_{-}\right\rangle$(using monotone convergence for the last equality), and this quantity is finite. Hence, $V_{\lambda}$ satisfies (1.6) and (see below (2.11)) $\Lambda\left(V_{\lambda}\right)<\infty$. So, by the first part of the proof,

$$
\Lambda\left(V_{\lambda}\right)=\Gamma\left(V_{\lambda}\right)(<\infty), \text { for } 0<\lambda \leq 1
$$

By monotone convergence in (2.3), we see that

$$
\lim _{\lambda \downarrow 0} \Lambda\left(V_{\lambda}\right)=\Lambda(V) .
$$

On the other hand, by (2.4) and (2.12), $\Gamma\left(V_{\lambda}\right)$ is a supremum of affine functions of $\lambda \in$ $[0,1]$, which is finite when $\lambda=1$ (since $V_{\lambda=1} \leq 0$ ) and when $\lambda=0$ (by assumption). Hence, it is a convex, lower semi-continuous, finite function on $[0,1]$, which is therefore continuous, so that

$$
\lim _{\lambda \rightarrow 0} \Gamma\left(V_{\lambda}\right)=\Gamma(V)
$$

This implies that (2.5) holds and completes the proof of Theorem 2.2.

Remark 2.3. The proof of Theorem 2.2 is easily adapted to the case of continuous time random interlacements on a transient weighted graph $E$. In this set-up, see for instance Section 1 of [30], one has a countable, locally finite, connected graph, with vertex set $E$, endowed with non-negative symmetric weights $c_{x, y}=c_{y, x}$, for $x, y \in E$, which are positive exactly when $x, y$ are distinct and $\{x, y\}$ is an edge of the graph. The induced continuous time random walk is assumed to be transient. It has exponential holding times of parameter 1 , and its discrete skeleton has transition probability

$$
p_{x, y}=\frac{c_{x, y}}{\lambda_{x}}, \text { where } \lambda_{x}=\sum_{z \in E} c_{x, z}, \text { for } x, y \in E .
$$

The continuous time random interlacements on the weighted graph can now be defined as a Poisson point process on a space of doubly-infinite $E$-valued trajectories, tending to infinity at plus and minus infinite times, marked by their duration at each step, modulo time-shift, see Section 1 of [30]. The field of occupation-times at level $u \geq 0$ in $x \in E$ corresponds to

$$
\begin{array}{r}
L_{x, u}(\omega)=\frac{1}{\lambda_{x}} \times \text { the total duration spent at } x \text { by trajectories modulo } \\
\text { time-shift, with label at most } u \text { in the cloud } \omega
\end{array}
$$

(durations of successive steps of a trajectory are described by independent exponential variables of parameter 1 , but occupation times at $x$ get rescaled by $\lambda_{x}^{-1}$ ).

In this set-up, one introduces for $V: E \rightarrow \mathbb{R}$, finitely supported

$$
\begin{aligned}
& \Lambda(V)= \frac{1}{u} \overline{\mathbb{E}}\left[\exp \left\{\sum_{x \in E} L_{x, u} V(x)\right\}\right] \text { (this does not depend on } u>0 \text { by the } \\
& \text { corresponding calculation to }(2.1) \text { ), and } \\
& \Gamma(V)=\langle V, 1\rangle+\sup _{\varphi \in C_{c}(E)}\{2\langle V, \varphi\rangle+\langle V \varphi, \varphi\rangle-\mathcal{E}(\varphi, \varphi)\},
\end{aligned}
$$


where $\langle f, g\rangle$ stands for $\sum_{x \in E} f(x) g(x)$ whenever the sum converges absolutely, 1 denotes the constant function equal to 1 on $E, C_{c}(E)$ stands for the set of finitely supported functions on $E$, and $\mathcal{E}$ for the Dirichlet form

$$
\mathcal{E}(f, f)=\frac{1}{2} \sum_{x, y \in E} c_{x, y}(f(y)-f(x))^{2}(\in[0, \infty]) \text { for } f: E \rightarrow \mathbb{R} .
$$

Let us mention that one can also replace $C_{c}(E)$ in $(2.23)$ by the extended space of the Dirichlet form $\mathcal{E}$ (see Chapter $1 \S 5$ of [13]). The proof of Theorem 2.2 adapted to the present context yields

\section{Theorem 2.2'.}

$$
\Lambda(V)=\Gamma(V) \text {, for all finitely supported } V: E \rightarrow \mathbb{R}
$$

We now derive some corollaries of Theorem 2.2, which will play an important role in the next section. As above $(0.1), B$ is a closed box (i.e. a compact subset of $\mathbb{R}^{d}$ that is the product of $d$ possibly different non-degenerate compact intervals in $\mathbb{R}$ ), and $m_{B}$, as above (0.2), the restriction of Lebesgue measure to $B$. We write $L^{p}(B)$ as a shorthand for $L^{p}\left(B, d m_{B}\right)$, when $1 \leq p<\infty$. Given $\widetilde{\varphi} \in L^{2}(B)$, we introduce

$$
\widetilde{\mathcal{E}}_{B}(\widetilde{\varphi}, \widetilde{\varphi})=\inf _{\varphi \in L^{2}\left(\mathbb{R}^{d}\right)}\{\mathcal{E}(\varphi, \varphi) ; \varphi=\widetilde{\varphi} \text { a.e. on } B\},
$$

the so-called trace Dirichlet form on $B$, see [13], pp. 265, 266. We will often drop the subscript $B$, when this causes no ambiguity. One knows that $\widetilde{\mathcal{E}}_{B}(\widetilde{\varphi}, \widetilde{\varphi})$ is finite precisely when $\widetilde{\varphi}$ is a.e. equal to the restriction to $B$ of a quasi-continuous function $\bar{\varphi}$ in the extended Dirichlet space $\mathcal{F}_{e}$, and in this case $\varphi(y)=E_{y}\left[\bar{\varphi}\left(X_{H_{B}}\right), H_{B}<\infty\right]$ belongs to $\mathcal{F}_{e}$ and $\widetilde{\mathcal{E}}_{B}(\widetilde{\varphi}, \widetilde{\varphi})=\mathcal{E}(\varphi, \varphi)$.

Let us also note that $L_{+}^{1}(B) \stackrel{\text { def }}{=}\left\{f \in L^{1}(B) ; f \geq 0, m_{B^{-a}}\right.$.e. $\}$ is a closed convex subset of $L^{1}(B)$ (endowed with the norm topology).

Corollary 2.4. For $V \in L_{c}^{\infty}\left(\mathbb{R}^{d}\right)$ vanishing outside $B$, one has

$$
\begin{aligned}
& \Lambda(V)=\sup _{h \in L_{+}^{1}(B)}\left\{\int_{B} V h d m_{B}-\widetilde{\mathcal{E}}(\sqrt{h}-1, \sqrt{h}-1)\right\}, \text { and } \\
& h \in L_{+}^{1}(B) \longrightarrow \widetilde{\mathcal{E}}(\sqrt{h}-1, \sqrt{h}-1) \in[0,+\infty] \text { is a convex lower } \\
& \text { semi-continuous function. }
\end{aligned}
$$

Proof. We consider $V \in L_{c}^{\infty}\left(\mathbb{R}^{d}\right)$ vanishing outside $B$. Then, by (2.5) we have

$$
\begin{aligned}
\Lambda(V) & =\sup _{\varphi \in L^{2}\left(\mathbb{R}^{d}\right)}\left\{\int V d y+2 \int V \varphi d y+\int V \varphi^{2} d y-\mathcal{E}(\varphi, \varphi)\right\} \\
& =\sup _{\varphi \in L^{2}\left(\mathbb{R}^{d}\right)}\left\{\int V(1+\varphi)^{2} d y-\mathcal{E}(\varphi, \varphi)\right\} \\
& \left.\stackrel{(2.26)}{=} \sup _{\widetilde{\varphi} \in L^{2}(B)}\left\{\int_{B} V(1+\widetilde{\varphi})^{2} d m_{B}-\widetilde{\mathcal{E}}(\widetilde{\varphi}, \widetilde{\varphi})\right\} \text { (since } V=0 \text { outside } B\right) .
\end{aligned}
$$


Now, the function $\rho(u)=|1+u|-1$ satisfies $\rho(0)=0$ and $|\rho(u)-\rho(v)| \leq|u-v|$, for $u, v \in \mathbb{R}$, and by [13], pp. 4, 5, we have for $\widetilde{\varphi} \in L^{2}(B), \widetilde{\psi}=\rho(\widetilde{\varphi}), \widetilde{\mathcal{E}}(\widetilde{\psi}, \widetilde{\psi}) \leq \widetilde{\mathcal{E}}(\widetilde{\varphi}, \widetilde{\varphi})$, and $(1+\widetilde{\psi})^{2}=(1+\widetilde{\varphi})^{2}$. As a result, we find that

$$
\Lambda(V)=\sup \left\{\int V(1+\widetilde{\psi})^{2} d m_{B}-\widetilde{\mathcal{E}}(\widetilde{\psi}, \widetilde{\psi}) ; \widetilde{\psi} \in L^{2}(B), 1+\widetilde{\psi} \geq 0 \text { a.e. }\right\}
$$

(the above argument shows that $\Lambda(V)$ is smaller or equal to the right-hand side of (2.30), but by the last line of $(2.29), \Lambda(V)$ is also bigger or equal to the right-hand side of (2.30)).

Setting $h=(1+\widetilde{\psi})^{2}$, we obtain a bijection between $\left\{\widetilde{\psi} \in L^{2}(B) ; 1+\widetilde{\psi} \geq 0\right.$, a.e. $\}$, and $L_{+}^{1}(B)$, and the claim (2.27) follows. As for (2.28), it is proved by a similar argument as in ii), below (4.2.64), p. 135 of [11] (see also Theorem 6.2.1 and (1.3.18), (1.4.8) in $[13])$.

The next corollary brings us a step closer to the identification of what will be the rate function of the large deviation principle, which we derive in the next section. We tacitly identify the set $L^{\infty}(B)$ of bounded measurable function on $B$ with the set $\left\{V \in L_{c}^{\infty}\left(\mathbb{R}^{d}\right)\right.$; $V=0$ on $B^{c}$, and recall that (see below $\left.(0.1)\right) C(B)$ stands for the space of continuous functions on $B$ (identified with the set of functions vanishing outside $B$, with continuous restriction to $B)$.

Corollary 2.5. For $h \in L_{+}^{1}(B)$, one has

$$
\begin{aligned}
\widetilde{\mathcal{E}}_{B}(\sqrt{h}-1, \sqrt{h}-1) & =\sup _{V \in L^{\infty}(B)}\left\{\int V h d m_{B}-\Lambda(V)\right\} \\
& =\sup _{V \in C(B)}\left\{\int V h d m_{B}-\Lambda(V)\right\} .
\end{aligned}
$$

Proof. Since $L^{\infty}\left(B, d m_{B}\right)$ is the dual of $L^{1}\left(B, d m_{B}\right)$, the first equality follows from (2.27), (2.28) and the duality formula in Theorem 2.2.15, p. 55 of [11], or Lemma 4.5.8, p. 152 of [10], together with the fact we can replace $L^{\infty}\left(B, d m_{B}\right)$ by $L^{\infty}(B)$, since the quantity under the supremum in the right-hand side of the first equality coincides for $V$ and $V^{\prime}$, if $V=V^{\prime}, m_{B}$-a.e. (recall (1.24)).

The quantity on the last line of (2.31) is obviously smaller or equal to the right-hand side of the first equality. Fix $h$ in $L_{+}^{1}(B)$. Our claim will thus follow, once we show that for any $V \in L^{\infty}(B)$ with $\Lambda(V)<\infty$ and $\varepsilon>0$, one can find $W \in C(B)$ such that

$$
\int W h d m_{B}-\Lambda(W) \geq \int V h d m_{B}-\Lambda(V)-\varepsilon .
$$

We thus pick $r>\frac{d}{2}$, as in Lemma 1.3, and choose $V_{n} \in C(B)$ (extended to be equal to 0 on $B^{c}$ ) such that

$$
\left\{\begin{aligned}
\text { i) } & \left\|V_{n}-V\right\|_{L^{r}\left(\mathbb{R}^{d}\right)} \rightarrow 0 \\
\text { ii) } & V_{n} \rightarrow V \text { a.e., } \\
\text { iii) } & \left\|V_{n}\right\|_{L^{\infty}\left(\mathbb{R}^{d}\right)} \leq\|V\|_{L^{\infty}\left(\mathbb{R}^{d}\right)} .
\end{aligned}\right.
$$


The construction of such a sequence can be performed as follows: one first multiplies $V$ by the indicator function of a slightly smaller concentric box in $B$, and then uses convolution by a smoothing kernel to construct a sequence $V_{n}$ of continuous functions vanishing on $B^{c}$, for which i) and iii) hold. One obtains ii) by extracting a suitable subsequence. Then Lemma 1.3 and (2.2) ensure that $\Lambda\left(V_{n}\right) \underset{n}{\longrightarrow} \Lambda(V)$, and moreover $\int V_{n} h d m_{B} \underset{n}{\longrightarrow} \int V h d m_{B}$, by dominated convergence. The claim (2.32) follows, and Corollary 2.5 is proved.

\section{Large deviations for occupation-time profiles of Brownian interlacements}

In this section, we derive a large deviation principle for the occupation-time profile of Brownian interlacements at level $\alpha>0$ in a box $L B$, as $L \rightarrow \infty$, cf. Corollary 3.3. Due to the scaling property (1.25) of $\mathcal{L}_{\alpha}$, this fixed level, large space problem (i.e. $\alpha>0$ fixed, and $L \rightarrow \infty$ ) is converted into a fixed space, large level problem (i.e. $L$ fixed, and $\alpha \rightarrow \infty$ ), which can be handled via general Cramér theory, see Chapter $6 \S 1$ of [10], or Chapter 3 of [11], making use of subadditivity, see Theorem 3.2. The identification of the rate function relies heavily on the results of the previous section, in particular on Corollary 2.5.

Given a closed box $B$ in $\mathbb{R}^{d}$ (see above $(0.1)$ ), we endow the space $M(B)$ of finite signed measures on $B$ with the weak topology generated by $C(B)$ (the space of continuous functions on $B$ ). The set $M_{+}(B)$ (of positive measures on $B$ ) is a closed convex subset of $M(B)$. We introduce the function on $M_{+}(B)$ (see (2.3) for notation):

$$
I_{B}(\mu)=\sup _{V \in C(B)}\left\{\int V d \mu-\Lambda(V)\right\} \in[0, \infty], \text { for } \mu \in M_{+}(B),
$$

and when there is no ambiguity, we simply write $I(\cdot)$ in place of $I_{B}(\cdot)$.

As we now see, $I_{B}(\cdot)$ is closely related to $I_{v}(\cdot), v>0$, in $(0.3)$.

Lemma 3.1. ( $B \subseteq B^{\prime}$ closed boxes in $\left.\mathbb{R}^{d}\right)$

$I_{B}(\cdot)$ is a convex, good rate function (i.e., it is convex, lower semi-continuous, and has compact level sets).

$I_{B}(\mu) \leq I_{B^{\prime}}\left(\mu^{\prime}\right)$ if $\mu$ is the restriction to $B$ of $\mu^{\prime} \in M_{+}\left(B^{\prime}\right)$.

$I_{B}(\mu)=+\infty$, if $\mu \in M_{+}(B)$ is not absolutely continuous with respect to $m_{B}$

$=\inf \left\{\frac{1}{2} \int_{\mathbb{R}^{d}}|\nabla \varphi|^{2} d y ; \varphi \in H^{1}\left(\mathbb{R}^{d}\right), \varphi=\left(\frac{d \mu}{d m_{B}}\right)^{\frac{1}{2}}-1\right.$ a.e. on $\left.B\right\}$,

if $\mu$ is absolutely continuous with respect to $m_{B}$.

$$
I_{v}(\cdot)=v I_{B}\left(\frac{\cdot}{v}\right), \text { for } v>0(\text { see }(0.3) \text { for notation })
$$


Proof. We begin with (3.2). The convexity and lower semi-continuity are direct consequences of (3.1). Moreover, when $\lambda>0$ is small enough so that $\lambda\left\|G 1_{B}\right\|_{L^{\infty}\left(\mathbb{R}^{d}\right)}<1$, we know that $\Lambda\left(\lambda 1_{B}\right)<\infty$, see (1.26), and hence,

$$
I(\mu) \geq \lambda\left\langle\mu, 1_{B}\right\rangle-\Lambda\left(\lambda 1_{B}\right), \text { for } \mu \in M_{+}(B) .
$$

Therefore, for any $M \geq 0,\left\langle\mu, 1_{B}\right\rangle$ remains bounded on $\left\{\mu \in M_{+}(B) ; I(\mu) \leq M\right\}$, which is therefore a compact subset of $M_{+}(B)$ (we already know it is closed by lower semicontinuity of $I$ ). This proves (3.2).

We now turn to (3.4). The second line of (3.4) is an immediate consequence of Corollary 2.5 and (2.26). To complete the proof of (3.4), it thus suffices to show that $I(\mu)=\infty$ when $\mu$ is not absolutely continuous with respect to $m_{B}$.

Indeed, for such a measure $\mu$, we can find a compact subset $K$ of $B$ such that $\mu(K)>$ 0 , but $m(K)=0$. Then, consider for $n \geq 1, W_{n}(\cdot)=(1-n d(\cdot, K))_{+} \in C(B)$ (we set $W_{n}$ equal to zero on $B^{c}$ ), so that $W_{n}$ is $[0,1]$-valued, takes the values 1 on $K$ and vanishes outside the open $\frac{1}{n}$-neighborhood of $K$ in $B$. Thus, choosing $r>\frac{d}{2}$, we see that $\delta_{n}=\left\|W_{n}\right\|_{L^{r}\left(\mathbb{R}^{d}\right)} \underset{n}{\longrightarrow} 0$, and setting $V_{n}=\delta_{n}^{-\frac{1}{2}} W_{n}$, it follows by Lemma 1.3 and (2.2) that $\Lambda\left(V_{n}\right) \underset{n}{\longrightarrow} 0$. As a consequence, we see that

$$
I(\mu) \stackrel{(3.1)}{\geq} \int V_{n} d \mu-\Lambda\left(V_{n}\right) \geq \delta_{n}^{-\frac{1}{2}} \mu(K)-\Lambda\left(V_{n}\right) \underset{n}{\longrightarrow}+\infty .
$$

This shows that $I(\mu)=\infty$ and completes the proof of (3.4).

We now prove (3.3). Without loss of generality, we can assume that $I_{B^{\prime}}\left(\mu^{\prime}\right)<\infty$. By (3.4) this implies that $\mu^{\prime}$ is absolutely continuous with respect to $m_{B^{\prime}}$, and hence $\mu$ is absolutely continuous with respect to $m_{B}$. The inequality $I_{B}(\mu) \leq I_{B}\left(\mu^{\prime}\right)$ is now a direct consequence of (3.4). This proves (3.3). Finally, (3.5) is immediate by comparison of (3.4) and (0.3).

We denote by $\mathcal{L}_{\alpha, B}$ the restriction of the (random) Radon measure $\mathcal{L}_{\alpha}$ on $\mathbb{R}^{d}$ to the Borel subsets of $B$. The main step towards the large deviation principle for $\nu_{L, \alpha}$, with $\alpha>0$ fixed and $L \rightarrow \infty$, see (0.5), is a large deviation principle for $\frac{1}{\alpha} \mathcal{L}_{\alpha, B}$, as $\alpha \rightarrow \infty$, which we derive in Theorem 3.2 below. We use general Cramér theory, see Theorem 6.1.3, p. 252 of [10] (with $\chi=M(B)$ and $\mathcal{E}=M_{+}(B)$, in the notation of [10]).

Theorem 3.2. (B a closed box)

$$
\text { As } \alpha \rightarrow \infty \text {, the laws under } \mathbb{P} \text { of } \frac{1}{\alpha} \mathcal{L}_{\alpha, B} \text { on } M_{+}(B) \text { satisfy a large deviation }
$$
principle at speed $\alpha$, with the convex good rate function $I_{B}$ from (3.1).

Moreover, for any open convex subset $O$ of $M(B)$,

$$
\lim _{\alpha \rightarrow \infty} \frac{1}{\alpha} \log \mathbb{P}\left[\frac{1}{\alpha} \mathcal{L}_{\alpha, B} \in O\right]=-\inf \left\{I_{B}(\mu) ; \mu \in O \cap M_{+}(B)\right\}
$$

(with $\inf \phi=\infty$, by convention). 
Proof. For any open convex subset $O$ of $M(B)$, the function

$$
f_{O}(\alpha)=-\log \mathbb{P}\left[\frac{1}{\alpha} \mathcal{L}_{\alpha, B} \in O\right] \in[0,+\infty], \text { for } \alpha>0,
$$

is subadditive (since $\mathcal{L}_{\alpha, B}$ is an $M_{+}(B)$-valued Lévy-process). When $O \cap M_{+}(B)=\phi$, then $f_{O}$ is identically infinite. Otherwise, when $O \cap M_{+}(B) \neq \phi$, then, as we now explain, for $\varepsilon \in(0,1]$ small,

$$
\sup _{1 \leq \alpha \leq 1+\varepsilon} f_{O}(\alpha)<\infty
$$

Indeed, for small $\varepsilon>0$, there is a positive $\mathbb{P}$-probability that exactly one trajectory of the interlacement at level 1 enters $B$ (i.e. $\mu_{B, \alpha=1}=1$, in the notation of (1.20)) and that $\mathcal{L}_{1, B} \in \alpha O$, for all $1 \leq \alpha \leq 1+\varepsilon$ (this can be arranged, using the fact that $O$ is open, $O \cap M_{+}(B) \neq \phi$, with the help of the support theorem for the Wiener measure, and the observation that once reaching distance 1 from $B$, a Brownian trajectory has a non-degenerate probability of never returning to $B$ ). Since $\mathcal{L}_{1+\varepsilon, B}-\mathcal{L}_{1, B}$ is independent from $\mathcal{L}_{1, B}$, and vanishes with positive probability, we see that on an event of positive $\mathbb{P}$-probability $\frac{1}{\alpha} \mathcal{L}_{\alpha, B} \in O$, for $1 \leq \alpha \leq 1+\varepsilon$. This is more than enough to prove (3.11).

Hence, by Lemma 4.2.5, p. 112 of [11], we see that when $O \cap M_{+}(B) \neq \phi$,

$$
\lim _{\alpha \rightarrow \infty} \frac{1}{\alpha} f_{O}(\alpha) \text { exists and is finite (and equals } \inf _{\alpha \geq \alpha_{0}} \frac{f_{O}(\alpha)}{\alpha} \text { for large } \alpha_{0} \text { ). }
$$

Moreover, choosing $\lambda$ as above (3.6), the Chebyshev Inequality and (1.26) imply that

$$
\mathbb{P}\left[\mathcal{L}_{\alpha}(B) \geq \alpha M\right] \leq \exp \left\{-\alpha\left(\lambda M-\Lambda\left(\lambda 1_{B}\right)\right)\right\}, \text { for } M>0, \alpha>0 .
$$

Since $\left\{\mu \in M_{+}(B) ; \mu(B) \leq M\right\}$ is compact for each $M>0$, and $\lambda M-\Lambda\left(\lambda 1_{B}\right) \underset{M}{\longrightarrow} \infty$, the exponential tightness of the laws of $\frac{1}{\alpha} \mathcal{L}_{\alpha, B}$ follows. Thus, by Theorem 4.1.11, p. 120 of [10], and p. 8 of the same reference, we see that, as $\alpha \rightarrow \infty$, the laws of $\frac{1}{\alpha} \mathcal{L}_{\alpha, B}$ satisfy a large deviation principle at speed $\alpha$. Restricting $\alpha$ to integer values, it follows from Theorem 6.1.3, p. 252 of [10] (Assumption 6.1.2 is straightforward to check in our set-up) that the rate function of the above large deviation principle coincides with $I_{B}$ in (3.1) and that the limit in (3.12) coincides with the right-hand side of (3.9) (when $O \cap M_{+}(B)=\phi$, both members of (3.12) equal $+\infty$ ). This concludes the proof of Theorem 3.2.

The large deviation principle for the profile $\nu_{L, \alpha}$ of the occupation-time measure of Brownian interlacements at level $\alpha$ in a large box $L B$ is now a direct consequence of Theorem 3.2 and the scaling property (1.25). We refer to (0.3), (0.5), and above (0.1) for notation.

Corollary 3.3. (Large deviation principle for the profile, $B$ a closed box, $\alpha>0$ )

As $L \rightarrow \infty$, the laws under $\mathbb{P}$ of $\nu_{L, \alpha}$ on $M_{+}(B)$ satisfy a large deviation principle with speed $L^{d-2}$ and rate function $I_{\alpha}$.

Moreover, for any open convex subset $O$ of $M(B)$,

$$
\lim _{L \rightarrow \infty} \frac{1}{L^{d-2}} \log \mathbb{P}\left[\nu_{L, \alpha} \in O\right]=-\inf \left\{I_{\alpha}(\mu) ; \mu \in O \cap M_{+}(B)\right\}
$$

(with inf $\phi=+\infty$ by convention). 
Proof. By the scaling property (1.25), we see that $\nu_{L, \alpha}$ has the same law as $\widetilde{\nu}_{L}=$ $\frac{1}{L^{d-2}} \mathcal{L}_{\alpha L^{d-2}, B}$, for any $L \geq 1$. Our claims are now direct consequences of Theorem 3.2.

\section{The discrete space set-up}

In this section, we introduce some additional notation concerning continuous time random interlacements on $\mathbb{Z}^{d}, d \geq 3$. We recall the scaling limit relating the discrete space occupation-times to the occupation-time measure of Brownian interlacements established in Theorem 3.2 of [31], see (4.4) below. Further, as a preparation to the large deviation principle for $\rho_{N, u}$ (see $(0.1)$ ), which we derive in the next section, we collect here the statements corresponding to Theorem 2.2' and Corollaries 2.4, 2.5 in the present set-up.

Given $N \geq 1$, we introduce the scaled lattice

$$
\mathbb{L}_{N}=\frac{1}{N} \mathbb{Z}^{d}\left(\subseteq \mathbb{R}^{d}\right)
$$

and $B$ being a closed box (see above $(0.1)$ ), we set

$$
B_{N}=B \cap \mathbb{L}_{N}
$$

For functions $f, h$ on $\mathbb{L}_{N}$ such that $\sum_{y \in \mathbb{L}_{N}}|f(y) h(y)|<\infty$, we write

$$
\langle f, h\rangle_{\mathbb{L}_{N}}=\frac{1}{N^{d}} \sum_{y \in \mathbb{L}_{N}} f(y) h(y)
$$

and we introduce the spaces $L^{p}\left(\mathbb{L}_{N}\right), 1 \leq p<\infty$, and their corresponding norms in a similar manner.

We refer to [30] for the precise construction of continuous time random interlacements on $\mathbb{Z}^{d}$ (with $d \geq 3$ ). As mentioned in the introduction, we denote by $(\bar{\Omega}, \overline{\mathcal{A}}, \overline{\mathbb{P}}$ ) the canonical space on which they are constructed, and by $\left(L_{x, u}\right)_{x \in \mathbb{Z}^{d}}$ the field of occupationtimes of random interlacements at level $u \geq 0$. Fixing $u \geq 0$, this is a stationary field on $\mathbb{Z}^{d}$, and by the same proof as for $(2.7)$ of $[28]$, it is ergodic. One knows that $\overline{\mathbb{E}}\left[L_{x, u}\right]=u$, for $x \in \mathbb{Z}^{d}, u \geq 0$, so by the ergodic theorem, see Theorem 2.8, p. 205 of [16], one sees that

$$
\text { for } u \geq 0, \overline{\mathbb{P}} \text {-a.s., } \frac{1}{N^{d}} \sum_{y \in \mathbb{L}_{N}} L_{N y, u} \delta_{y} \text { converges vaguely to } u d y \text {, as } N \rightarrow \infty
$$

(and $\rho_{N, u}$ converges weakly to $u m_{B}$, in the notation of $(0.1)$ ).

The occupation-time measure of Brownian interlacements can be expressed as a scaling limit of the occupation times of random interlacements on $\mathbb{Z}^{d}$. Namely, one know by Theorem 3.2 of [31] that for $\alpha \geq 0$, as $N \rightarrow \infty$,

$$
\mathcal{L}_{N, \alpha} \stackrel{\text { def }}{=} \frac{1}{d N^{2}} \sum_{y \in \mathbb{L}_{N}} L_{N y, d \alpha N^{2-d}} \delta_{y} \text { converges in distribution to } \mathcal{L}_{\alpha}
$$

(we endow the set of Radon measures on $\mathbb{R}^{d}$ with the topology of vague convergence). 
For $N \geq 1$ and $V: \mathbb{L}_{N} \rightarrow \mathbb{R}$ with finite support, we introduce

$$
\Lambda_{N}(V)=\log \overline{\mathbb{E}}\left[e^{\left\langle\mathcal{L}_{N, 1}, V\right\rangle}\right] \in(-\infty,+\infty],
$$

and note that

$$
\Lambda_{N}(V)=\frac{d}{N^{d-2}} \log \overline{\mathbb{E}}\left[e^{\frac{N^{d-2}}{d}\left\langle\rho_{N, u=1}, V\right\rangle}\right] \text {, when } V \text { vanishes outside } B_{N}
$$

(where we used (0.1) and the fact that the right-hand side of (2.22) does not depend on $u)$.

To state the identity corresponding to Theorem 2.2 ' in the present set-up, we define, for $N \geq 1$ and $V: \mathbb{L}_{N} \rightarrow \mathbb{R}$ finitely supported,

$$
\Gamma_{N}(V)=\langle V, 1\rangle_{\mathbb{L}_{N}}+\sup _{\varphi \in L^{2}\left(\mathbb{L}_{N}\right)}\left\{2\langle V, \varphi\rangle_{\mathbb{L}_{N}}+\langle V \varphi, \varphi\rangle_{\mathbb{L}_{N}}-\mathcal{E}_{N}(\varphi, \varphi)\right\},
$$

where for $\varphi \in L^{2}\left(\mathbb{L}_{N}\right)$

$$
\mathcal{E}_{N}(\varphi, \varphi)=\frac{1}{2 N^{d-2}} \sum_{y \sim y^{\prime} \text { in } \mathbb{L}_{N}} \frac{1}{2}\left(\varphi\left(y^{\prime}\right)-\varphi(y)\right)^{2} \in[0, \infty]
$$

$\left(y \sim y^{\prime}\right.$ means that $y$ and $y^{\prime}$ are neighbors in $\mathbb{L}_{N}$, i.e. $\left.\left|y-y^{\prime}\right|=\frac{1}{N}\right)$.

We also keep the notation $\mathcal{E}_{N}(\varphi, \varphi)$ when $\varphi$ belongs to the extended space of the Dirichlet form $\mathcal{E}_{N}$ (corresponding to functions on $\mathbb{L}_{N}$ that are pointwise limits of an $\mathcal{E}_{N^{-}}$ Cauchy sequence of finitely supported functions on $\mathbb{L}_{N}$ ). After proper normalization (the $V$ in Theorem 2.2' corresponds to $\left.\frac{1}{d N^{2}} V(\dot{\bar{N}})\right)$, Theorem 2.2' now yields

Theorem 4.1. $(N \geq 1)$

$$
\Lambda_{N}(V)=\Gamma_{N}(V), \text { for all } V: \mathbb{L}_{N} \rightarrow \mathbb{R} \text { with finite support. }
$$

Before stating the corollary corresponding to Corollaries 2.4, 2.5 in the present set-up, we define for $\psi: B_{N} \rightarrow \mathbb{R}$,

$$
\widetilde{\mathcal{E}}_{N}(\psi, \psi)=\inf \left\{\mathcal{E}_{N}(\varphi, \varphi) ; \varphi \in L^{2}\left(L_{N}\right), \varphi=\psi \text { on } B_{N}\right\} .
$$

Denoting by $P_{y}^{N}$, for $N \geq 1, y \in \mathbb{L}_{N}$, the canonical law of the simple random walk on $\mathbb{L}_{N}$ with exponential holding times of parameter $N^{2}$, starting at $y \in \mathbb{L}_{N}$, and using similar notation for the canonical process, the entrance times, and the exit times, as described at the beginning of Section 1, one knows, as below (2.26), that

$$
\widetilde{\mathcal{E}}_{N}(\psi, \psi)=\mathcal{E}_{N}(\varphi, \varphi) \in[0, \infty),
$$

where $\varphi(y)=E_{y}^{N}\left[\psi\left(X_{H_{B_{N}}}\right), H_{B_{N}}<\infty\right]$, for $y \in \mathbb{L}_{N}$, is harmonic outside $B_{N}$, tends to zero at infinity, and belongs to the extended Dirichlet space of $\mathcal{E}_{N}$ (with $H_{B_{N}}$ the entrance time in $\left.B_{N}\right)$.

Similarly to Corollaries 2.4 and 2.5 (but in a much simpler fashion), we have 
Corollary 4.2. $(N>1)$

When $V: \mathbb{L}_{N} \rightarrow \mathbb{R}$ vanishes outside $B_{N}$

$$
\Lambda_{N}(V)=\sup _{h \geq 0 \text { on } B_{N}}\left\{\langle V, h\rangle_{\mathbb{L}_{N}}-\widetilde{\mathcal{E}}_{N}(\sqrt{h}-1, \sqrt{h}-1)\right\} \in[0, \infty],
$$

and for $h: B_{N} \rightarrow \mathbb{R}_{+}$(extended to be equal to 0 outside $B_{N}$ )

$$
\widetilde{\mathcal{E}}_{N}(\sqrt{h}-1, \sqrt{h}-1)=\sup _{V=0 \text { on } \mathbb{L}_{N} \backslash B_{N}}\left\{\langle V, h\rangle_{\mathbb{L}_{N}}-\Lambda_{N}(V)\right\} .
$$

\section{Large deviations for occupation-time profiles of ran- dom interlacements}

The main object of this section is to prove a large deviation principle for the occupationtime profile $\rho_{N, u}$, see $(0.1)$, of continuous time random interlacements on $\mathbb{Z}^{d}$ at level $u$, when $N \rightarrow \infty$, cf. Theorem 5.8. Subadditivity is an important ingredient in our proof, see Proposition 5.2 and Corollary 5.3. The lower bound in the large deviation principle appears in Theorem 5.4. It relies on Corollary 5.3, and on the combination of the large deviation principle for the occupation-time profile of Brownian interlacements proved in Section 3, and the scaling limit result (4.5) proved in [31], see also Lemma 5.1 below. The upper bound appears in Theorem 5.5. It relies on Corollary 5.3 and on Proposition 5.6, which provides an asymptotic lower bound for a sequence of discrete variational problems, in the spirit of $\Gamma$-convergence, see Proposition 7.2, p. 68 of [9].

We pick $u>0$ and a closed box $B$ (see above (0.1)). The space $M(B)$ is equipped with the weak topology generated by $C(B)$, as explained in Section 3. We consider $\nu \in M_{+}(B)$, a finite collection $f_{\ell} \in C(B), 1 \leq \ell \leq K$, with $f_{1}=1_{B}$, and a number $\delta>0$. We define the convex open subset $A$ of $M_{+}(B)$ consisting of positive measures on $B$ with integrals with respect to $f_{\ell}, 1 \leq \ell \leq K, \delta$-close to the corresponding integrals with respect to $\nu$, and we denote by $O$ the homothetic image of $A$ with ratio $u$ :

$$
A=\left\{\rho \in M_{+}(B) ;\left|\left\langle\rho, f_{\ell}\right\rangle-\left\langle\nu, f_{\ell}\right\rangle\right|<\delta, \text { for } 1 \leq \ell \leq K\right\}, \text { and } O=u A .
$$

We use the shorthand notation $c(A)$ to denote a positive constant, which depends on $d, B, \nu, K,\left(f_{\ell}\right)_{1 \leq \ell \leq K}, \delta$. The collection of sets $A$ (or $O$ ) above constitutes a base for the relative topology on $M_{+}(B)$ (viewed as a subset of $M(B)$ ).

We also define for $N \geq 1, t \geq 0$ (see (4.5) for notation)

$$
\widetilde{\mathcal{L}}_{N, t}=\text { the restriction of } \mathcal{L}_{N, t} \text { to Borel subsets of } B
$$

(a random element of $M_{+}(B)$ ). We now state a consequence of (4.5).

Lemma 5.1. $(\alpha \geq 0)$

(5.3) As $N \rightarrow \infty, \widetilde{\mathcal{L}}_{N, \alpha}$ converges in distribution to $\mathcal{L}_{\alpha, B}$ (see above (3.8) for notation) 
Proof. By (4.5) and the continuous mapping theorem, see Theorem 5.1, p. 30 of [2], it suffices to show that (denoting the set of Radon measures on $\mathbb{R}^{d}$ by $\mathcal{M}_{+}\left(\mathbb{R}^{d}\right)$ ):

the set of continuity points of the map $\rho \in \mathcal{M}_{+}\left(\mathbb{R}^{d}\right) \rightarrow \widetilde{\rho} \in M_{+}(B)$, where $\tilde{\rho}$ is

the restriction of $\rho$ to Borel subsets of $B$, has full measure under the law of $\mathcal{L}_{\alpha}$.

To see this point, note that by (1.24), for a.e. $\rho$ under the law of $\mathcal{L}_{\alpha}$, one has $\rho(\partial B)=0$. Hence, for any such $\rho$, for any $V \in C(B)$ (extended as 0 outside $B$ ), and any sequence $\rho_{n}$ converging vaguely to $\rho$, one has $\left\langle\rho_{n}, V\right\rangle \underset{n}{\longrightarrow}\langle\rho, V\rangle$. This proves that $\widetilde{\rho}_{n}$ converges weakly to $\widetilde{\rho}$ in $M_{+}(B)$ and (5.4) follows.

We then introduce

$$
f_{N, A}(t)=-\log \overline{\mathbb{P}}\left[\frac{1}{t} \widetilde{\mathcal{L}}_{N, t} \in A\right], \text { for } t>0, N \geq 1 .
$$

Since $t \rightarrow \widetilde{\mathcal{L}}_{N, t}$ has independent, stationary, increments, and $A$ is convex,

$$
\text { for each } N \geq 1, f_{N, A}(\cdot) \text { is subadditive. }
$$

The next proposition collects some important bounds on $\overline{\mathbb{P}}\left[\frac{1}{t} \mathcal{L}_{N, t} \in A\right]$, which exploit subadditivity. It comes as a step towards Corollary 5.3 below. We recall the convention on constants stated below (5.1).

Proposition 5.2. When $N \geq c_{0}(A)$, then for $t_{1} \geq c(A)$ and $t \geq 2 t_{1}$, one has

$$
\exp \left\{-t \frac{f_{N, A}\left(t_{1}\right)}{t_{1}}-2 t_{1} c^{\prime}(A)\right\} \leq \overline{\mathbb{P}}\left[\frac{1}{t} \widetilde{\mathcal{L}}_{N, t} \in A\right] \leq \exp \left\{-t \lim _{s \rightarrow \infty} \frac{f_{N, A}(s)}{s}\right\}
$$

(and the limit in the rightmost term of (5.7) exists and is finite).

Proof. We first show that for some $\varepsilon_{A}=1 / q_{A}$, where $q_{A}$ is some positive integer depending on $A$ (with a similar meaning as below (5.1)) and for some $N_{0}(A)$

$$
\sup _{N \geq N_{0}(A)} \sup _{t \in\left[1,1+\varepsilon_{A}\right]} f_{N, A}(t)=M<\infty .
$$

To see this point, we introduce $A^{\prime}$ defined as $A$ in (5.1) with $\delta$ replaced by $\frac{\delta}{2}$. By (5.3) and (3.11), we know that $\lim \inf _{N} \overline{\mathbb{P}}\left[\widetilde{\mathcal{L}}_{N, 1} \in A^{\prime}\right] \geq \mathbb{P}\left[\mathcal{L}_{1, B} \in A^{\prime}\right]>0$. Hence, for $N \geq N_{0}(A)$, $\overline{\mathbb{P}}\left[\widetilde{\mathcal{L}}_{N, 1} \in A^{\prime}\right] \geq c(A)$. Then, the probability that no trajectory of the interlacement with label in $\left(d N^{2-d}, d(1+\varepsilon) N^{2-d}\right]$ enters $N B_{N}$ is equal to $e^{-d \varepsilon N^{2-d} \operatorname{cap}_{\mathbb{Z}^{d}}\left(N B_{N}\right)} \geq e^{-c(B) \varepsilon}$ (with $\operatorname{cap}_{\mathbb{Z}^{d}}(\cdot)$ the capacity on $\mathbb{Z}^{d}$, see for instance (1.57) of [28]). Such an event is independent under $\overline{\mathbb{P}}$ of $\left\{\widetilde{\mathcal{L}}_{N, 1} \in A^{\prime}\right\}$. When $\varepsilon=\varepsilon_{A}=1 / q_{A}$ with $q_{A}$ a large enough integer, so that the set of multiples by a scalar in $\left[\left(1+\varepsilon_{A}\right)^{-1}, 1\right]$ of a measure in $A^{\prime}$ is contained in $A$, on the intersection of these two events, one has $\frac{1}{t} \widetilde{\mathcal{L}}_{N, t}=\frac{1}{t} \widetilde{\mathcal{L}}_{N, 1} \in A$, for all $1 \leq t \leq 1+\varepsilon_{A}$. The claim (5.8) follows.

We can now apply Lemma 4.2.5, p. 112 of [11], and find that when $t_{1} \geq q_{A}$, then for $t \geq 2 t_{1}$ and $N \geq N_{0}(A)$, one has (with [.] denoting the integer part)

$$
f_{N, A}(t) \leq\left(\left[\frac{t}{t_{1}}\right]-1\right) f_{N, A}\left(t_{1}\right)+2 t_{1} M \leq t \frac{f_{N, A}\left(t_{1}\right)}{t_{1}}+2 t_{1} M .
$$


This proves the first inequality in (5.7).

As for the second inequality, we know by the same Lemma 4.2.5 of [11], that the limit in the rightmost term of (5.7) exists, is finite, and that moreover, for $t \geq q_{A}$,

$$
\lim _{s \rightarrow \infty} \frac{f_{N, A}(s)}{s} \leq \frac{f_{N, A}(t)}{t}
$$

This implies the second inequality in (5.7) (and one can choose $c(A)=q_{A}$ in (5.7)).

For fixed $N \geq 1$, the finite dimensional space of signed measures spanned by the basis $\frac{1}{N^{d}} \delta_{y}, y \in B_{N}$, is a closed subspace of $M(B)$, and the corresponding coordinates yield an homeomorphism with $\mathbb{R}^{B_{N}}$. The intersection of this space with $M_{+}(B)$ consists of linear combinations of $\frac{1}{N^{d}} \delta_{y}, y \in B_{N}$, with non-negative coefficients, and defines a closed convex subset of $M_{+}(B)$. The finite convex lower semi-continuous (it is actually continuous) function on $\mathbb{R}_{+}^{B_{N}}$ in (4.14), extended to be equal to $+\infty$ on the complement in $M_{+}(B)$ of its domain of definition, yields a convex rate function (i.e. a convex, lower semi-continuous, $[0,+\infty]$-valued function) denoted by

$$
\begin{aligned}
I_{N}(\mu) & =\widetilde{\mathcal{E}}_{N}(\sqrt{h}-1, \sqrt{h}-1), \text { if } \mu=\frac{1}{N^{d}} \sum_{y \in B_{N}} h(y) \delta_{y}, \text { with } h: B_{N} \rightarrow \mathbb{R}_{+}, \\
& =+\infty, \text { otherwise. }
\end{aligned}
$$

We can now state a consequence of Proposition 5.2. The difference lies in the rightmost inequality, which now involves the functional $I_{N}$. The next Corollary 5.3 encapsulates the sub-additivity lower and upper bounds, which we will respectively use in the proofs of Theorem 5.4 and of Theorem 5.5.

Corollary 5.3. (with $A$ as in (5.1))

When $N \geq N_{0}(A)$, for $t_{1} \geq c(A)$ and $t \geq 2 t_{1}$, one has

$$
\exp \left\{-t \frac{f_{N, A}\left(t_{1}\right)}{t_{1}}-2 t_{1} c^{\prime}(A)\right\} \leq \overline{\mathbb{P}}\left[\frac{1}{t} \widetilde{\mathcal{L}}_{N, t} \in A\right] \leq \exp \left\{-t \inf _{\rho \in A} I_{N}(\rho)\right\}
$$

Proof. Recall that $t \rightarrow \widetilde{\mathcal{L}}_{N, t}$ has independent, stationary increments. By Theorem 6.1.3, p. 252 of [10], and (4.6), (4.14), we know that for each fixed $N \geq 1$, the laws of $\frac{1}{n} \widetilde{\mathcal{L}}_{N, n}$ (on $M_{+}(B)$ ), as $n \rightarrow \infty$, satisfy a weak large deviation principle at speed $n$, with convex rate function $I_{N}$, see (5.10). Moreover, by the same reference, $\lim _{n}-\frac{f_{N, A}(n)}{n}=-\inf _{A} I_{N}$ $(\in[-\infty, 0])$. The comparison with (5.7) yields (5.11).

We can now derive the asymptotic lower bound.

Theorem 5.4. (Large deviation lower bound, $u>0, O$ as in (5.1))

$$
\frac{\lim }{N} \frac{1}{N^{d-2}} \log \overline{\mathbb{P}}\left[\rho_{N, u} \in O\right] \geq-\inf _{\mu \in O} \frac{1}{d} I_{u}(\mu) \text { (see (0.3) for notation). }
$$


Proof. We first use sub-additivity. We set $t=\frac{u}{d} N^{d-2}$, so that $\rho_{N, u}=\frac{u}{t} \widetilde{\mathcal{L}}_{N, t}$ (see $(0.1)$ and (4.5), (5.2)), we see by the first inequality of (5.11) that for any $t=\frac{u}{d} N^{d-2} \geq 2 t_{1} \geq c(A)$ and $N \geq N_{0}(A)$ (recall $\left.O=u A\right)$,

$$
\overline{\mathbb{P}}\left[\rho_{N, u} \in O\right] \stackrel{(5.1)}{=} \overline{\mathbb{P}}\left[\frac{1}{t} \widetilde{\mathcal{L}}_{N, t} \in A\right] \geq \exp \left\{-t \frac{f_{N, A}\left(t_{1}\right)}{t_{1}}-2 t_{1} c^{\prime}(A)\right\} .
$$

Hence, for any $t_{1} \geq c(A)$,

$$
\frac{\lim }{N} \frac{1}{N^{d-2}} \log \overline{\mathbb{P}}\left[\rho_{N, u} \in O\right] \geq-\frac{u}{d} \varlimsup_{N} \frac{f_{N, A}\left(t_{1}\right)}{t_{1}} .
$$

We now use convergence in law, and note that by Lemma 5.1

$$
\frac{\varliminf_{N}}{\mathbb{P}}\left[\widetilde{\mathcal{L}}_{N, t_{1}} \in t_{1} A\right] \geq \mathbb{P}\left[\mathcal{L}_{t_{1}, B} \in t_{1} A\right] .
$$

Taking logarithms (recall the notation from (5.5) and (3.10)), we find that

$$
-\varlimsup_{N} f_{N, A}\left(t_{1}\right) \geq-f_{A}\left(t_{1}\right) .
$$

We are now in position to use the key large deviation result from Section 3. Specifically, coming back to (5.13) we see that for $t_{1} \geq c(A)$

$$
\frac{\lim }{N} \frac{1}{N^{d-2}} \log \overline{\mathbb{P}}\left[\rho_{N, u} \in O\right] \geq-\frac{u}{d} \frac{f_{A}\left(t_{1}\right)}{t_{1}} \underset{t_{1} \rightarrow \infty}{\stackrel{(3.9)}{\longrightarrow}}-\frac{u}{d} \inf _{\rho \in A} I_{B}(\rho) \stackrel{(3.5)}{=}-\inf _{\mu \in O} \frac{1}{d} I_{u}(\mu) .
$$

This proves Theorem 5.4.

We now turn to the proof of the asymptotic upper bound. An argument based on the convergence of $\Lambda_{N}(V)$ to $\Lambda(V)$ (or on the asymptotic domination of $\Lambda_{N}(V)$ by $\Lambda(V)$ ), for all $V$ in $C(B)$ does not seem straightforward. Instead, we use a strategy, which is in the spirit of $\Gamma$-convergence for the functions $I_{N}$, see Proposition 5.6 below, and Proposition 7.2, p. 68 of [9]. Another possible, although somewhat indirect, route might be to use the isomorphism theorem of [30] and the large deviation principles on the empirical distribution functional of the Gaussian free field on $\mathbb{Z}^{d}$, proved in [3].

Theorem 5.5. (Large deviation upper bound, $u>0, O$ as in (5.1))

$$
\varlimsup_{N} \frac{1}{N^{d-2}} \log \overline{\mathbb{P}}\left[\rho_{N, u} \in O\right] \leq-\inf _{\mu \in \bar{O}} \frac{1}{d} I_{u}(\mu)
$$

(with $\bar{O}$ the closure of $O$ in $M_{+}(B)$ ).

Proof. We first exploit sub-additivity. Setting $t=\frac{u}{d} N^{d-2}$, we know by Corollary 5.3 that for $t \geq 2 c(A), N \geq N_{0}(A)$ (recall $\left.O=u A\right)$,

$$
\overline{\mathbb{P}}\left[\rho_{N, u} \in O\right] \stackrel{(5.1)}{=} \overline{\mathbb{P}}\left[\frac{1}{t} \widetilde{\mathcal{L}}_{N, t} \in A\right] \stackrel{(5.11)}{\leq} \exp \left\{-t \inf _{\rho \in A} I_{N}(\rho)\right\} .
$$

Hence, we find that

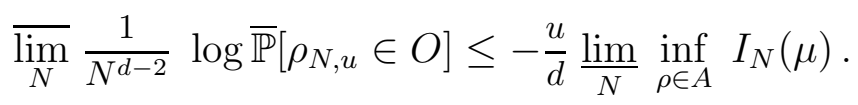

The proof of (5.15) relies on the crucial next proposition. 
Proposition 5.6. ( $K$ compact subset of $M_{+}(B)$ )

$$
\frac{\lim }{N} \inf _{\rho \in K} I_{N}(\rho) \geq \inf _{\rho \in K} I(\rho) \text { (see (3.1), (5.10) for notation). }
$$

Let us admit Proposition 5.6 for the time being, and first complete the proof of Theorem 5.5. By (5.16) and (5.17) (with $K=\bar{A}$ ), we see that

$$
\varlimsup_{N} \frac{1}{N^{d-2}} \log \overline{\mathbb{P}}\left[\rho_{N, u} \in O\right] \leq-\frac{u}{d} \inf _{\bar{A}} I \stackrel{(5.5)}{=}-\inf _{\bar{O}} \frac{1}{d} I_{u} .
$$

This proves Theorem 5.5 (conditionally on Proposition 5.6).

There remains to prove Proposition 5.6.

It may be useful at this point to provide an outline of its proof. In essence, assuming that the left-hand side of (5.17) is finite (otherwise (5.17) is obvious), we will consider a minimizing subsequence $\mu_{\ell}$ in $K\left(\subseteq M_{+}(B)\right)$ such that $I_{N_{\ell}}\left(\mu_{\ell}\right)$ tends to the left-hand side of (5.17) and $N_{\ell}$ tends to infinity. By the relationship between $I_{N_{\ell}}$ and $\mathcal{E}_{N_{\ell}}$ from (5.10) and (4.12), we will recast $I_{N_{\ell}}\left(\mu_{\ell}\right)$ as $\mathcal{E}_{N_{\ell}}\left(\varphi_{\ell}, \varphi_{\ell}\right)$, where $\varphi_{\ell}$ are functions on $\mathbb{L}_{N_{\ell}}$, harmonic outside $B_{N_{\ell}}$, tending to zero at infinity, with value at least -1 in $B_{N_{\ell}}$, and such that $\mu_{\ell}$ has density $1_{B_{N_{\ell}}}\left(1+\varphi_{\ell}\right)^{2} N_{\ell}^{-d}$ with respect to the counting measure on the scaled lattice $\mathbb{L}_{N_{\ell}}$. With the help of a cut-off lemma (see Lemma 5.7 below), we will replace the sequence $\left(\varphi_{\ell}\right)$ by a sequence $\left(\bar{\varphi}_{\ell}\right)$. In particular, $\bar{\varphi}_{\ell}$ will coincide with $\varphi_{\ell}$ on $B_{N_{\ell}}$, but will vanish outside a fixed compact set, independent of $\ell$, and $\mathcal{E}_{N_{\ell}}\left(\bar{\varphi}_{\ell}, \bar{\varphi}_{\ell}\right)$ will not be substantially bigger than $\mathcal{E}_{N_{\ell}}\left(\varphi_{\ell}, \varphi_{\ell}\right)$. We will then introduce a step function $\Phi_{\ell}$ on $\mathbb{R}^{d}$, constant on cubes of side-length $\frac{1}{N_{\ell}}$, which coincides with $\bar{\varphi}_{\ell}$ on $\mathbb{L}_{N_{\ell}}$. Making use of the controls on $\bar{\varphi}_{\ell}$ stemming from the Dirichlet form and the compact support of the functions $\Phi_{\ell}$, we will show that $\Phi_{\ell}, \ell \geq 1$, is relatively compact in $L^{2}\left(\mathbb{R}^{d}\right)$. We will extract a convergent subsequence to a compactly supported $\Phi$ in $L^{2}\left(\mathbb{R}^{d}\right)$, having value at least -1 on $B$, such that the measure $\mu=(1+\Phi)^{2} m_{B}$ belongs to $K$ (the compact subset of $M_{+}(B)$ in the statement of Proposition 5.6), and $I(\mu)$ is not substantially bigger than the left-hand side of (5.17). This will show that the infimum of $I$ over $K$ is smaller or equal to $\underline{\lim }_{N} \underline{\inf }_{K} I_{N}$ and conclude the proof of Proposition 5.6.

Proof of Proposition 5.6. We begin with a cut-off lemma for functions on $\mathbb{L}_{N}$, which are harmonic off $B_{N}$ and tend to 0 at infinity. This lemma, as mentioned above, will be an important ingredient when proving the relative compactness of a suitable nearly minimizing sequence we later construct.

We introduce $L_{B} \geq 1$ the smallest positive integer such that (see the beginning of Section 1 for notation)

$$
B \subseteq B_{\infty}\left(0, L_{B}\right),
$$

and for $R \geq 0$ integer, the closed box

$$
C_{R}=B_{\infty}\left(0, L_{B}(R+2)\right) \supseteq B,
$$

so that $d_{\infty}\left(B_{\infty}\left(0, L_{B}\right), \partial C_{R}\right) \geq L_{B}(R+1)$. For functions $\varphi$ defined on $\mathbb{L}_{N}$, we use, as a shorthand, the notation $\sup _{B} \varphi, \inf _{C_{R}} \varphi, \ldots$, in place of $\sup _{B \cap \mathbb{L}_{N}} \varphi, \inf _{C_{R} \cap \mathbb{L}_{N}} \varphi, \ldots$.

We are now ready to state and prove the cut-off lemma. The reader may choose to first skip its proof. 
Lemma 5.7. (Cut-off lemma, $R \geq 1$ integer)

Let $N \geq 1$, and $\varphi$ on $\mathbb{L}_{N}$ be harmonic outside $B_{N}$ and tend to 0 at infinity. There exists $\bar{\varphi}$ on $\mathbb{L}_{N}$ such that

$$
\left\{\begin{aligned}
& \text { i) } \bar{\varphi}=\varphi \text {, on } C_{R}, \\
& \text { ii) } \bar{\varphi}=0 \text { outside } C_{100 R}, \\
& \text { iii) } \mathcal{E}_{N}(\bar{\varphi}, \bar{\varphi}) \leq \mathcal{E}_{N}(\varphi, \varphi)\left(1+\frac{c(B)}{R^{d-2}}\right) .
\end{aligned}\right.
$$

Proof. To simplify notation, all constants in the proof implicitly depend on $d$ and $B$.

We let $\psi$ and $\gamma$ stand for the harmonic extensions of $\varphi_{+}=\max \{\varphi, 0\}$ and $\varphi_{-}=$ $\max \{-\varphi, 0\}$ outside $B_{N}$ (on $\mathbb{L}_{N} \backslash B_{N}$ ), which tend to 0 at infinity. Since $\widetilde{\mathcal{E}}_{N}$ in (4.11) is a Dirichlet form, we know that the restrictions $\widetilde{\varphi}, \widetilde{\varphi}_{+}, \widetilde{\varphi}_{-}$of $\varphi, \varphi_{+}, \varphi_{-}$to $B_{N}$ satisfy

$$
\begin{aligned}
\mathcal{E}_{N}(\psi, \psi) \stackrel{(4.12)}{=} \widetilde{\mathcal{E}}_{N}\left(\widetilde{\varphi}_{+}, \widetilde{\varphi}_{+}\right) \leq \widetilde{\mathcal{E}}_{N}(\widetilde{\varphi}, \widetilde{\varphi}) \stackrel{(4.12)}{=} \mathcal{E}_{N}(\varphi, \varphi) \text { and } \\
\mathcal{E}_{N}(\gamma, \gamma) \leq \mathcal{E}_{N}(\varphi, \varphi) \text { (in a similar fashion). }
\end{aligned}
$$

By the Harnack inequality and chaining (see Theorem 1.7.2, p. 42 of [17]), we have

$$
\max _{\partial C_{0}} \psi \leq c \min _{\partial C_{0}} \psi \text { and } \max _{\partial C_{0}} \gamma \leq c \min _{\partial C_{0}} \gamma
$$

(we recall that all constants in the proof of Lemma 5.7 implicitly depend on $d$ and $B$ ). Since $\psi$ is harmonic outside $C_{0}$ and tends to zero at infinity, it follows, by a stopping argument, that for $y$ in $\partial C_{R}, \psi(y)$ is smaller than the product of $\max _{\partial C_{0}} \psi$ with the probability for the walk on $\mathbb{L}_{N}$ starting at $y$ to reach $\partial C_{0}$. A similar bound holds for $\gamma$. By (5.22) and classical random walk estimates, we obtain

$$
\max _{\partial C_{R}} \psi \leq \frac{c}{R^{d-2}} \min _{\partial C_{0}} \psi \stackrel{\text { def }}{=} a_{R}, \max _{\partial C_{R}} \gamma \leq \frac{c}{R^{d-2}} \min _{\partial C_{0}} \gamma \stackrel{\text { def }}{=} b_{R} \text {, for } R \geq 1 .
$$

By estimates on the discrete gradient of harmonic functions in large balls, see Theorem 1.7.1, p. 42 of [17], we have for $R \geq 1, y \in \mathbb{L}_{N} \backslash C_{R}$ and $y^{\prime} \sim y$ in $\mathbb{L}_{N}$,

$$
\left|\psi\left(y^{\prime}\right)-\psi(y)\right| \leq \frac{c}{R^{d-2}} \frac{1}{R N} \min _{\partial C_{0}} \psi,\left|\gamma\left(y^{\prime}\right)-\gamma(y)\right| \leq \frac{c}{R^{d-2}} \frac{1}{R N} \min _{\partial C_{0}} \gamma .
$$

With a similar notation as below (4.11), we define for $R \geq 1$

$$
q_{R}(y)=P_{y}^{N}\left[H_{C_{R} \cap \mathbb{L}_{N}}>T_{C_{100 R} \cap \mathbb{L}_{N}}\right] \text {, for } y \in \mathbb{L}_{N},
$$

the probability for the walk on $\mathbb{L}_{N}$ starting at $y$ to exit $C_{100 R}$ before entering $C_{R}$. We then define

$$
\begin{aligned}
\bar{\varphi}(y) & =\varphi(y) \text { on } C_{R} \cap \mathbb{L}_{N}, \\
& =\psi(y) \wedge\left(a_{R} q_{R}(y)\right)-\gamma(y) \wedge\left(b_{R} q_{R}(y)\right), \text { on } \mathbb{L}_{N} \backslash C_{R}
\end{aligned}
$$

(note that the expressions on both lines are equal for $y \in \partial C_{R} \cap \mathbb{L}_{N}$ ). 
For simple random walk on $\mathbb{Z}^{d}$, the capacity of $\left(N C_{R}\right) \cap \mathbb{Z}^{d}$ relative to $\left(N C_{100 R}\right) \cap \mathbb{Z}^{d}$ is at most $c(N R)^{d-2}$, see for instance (5.26) of [27]. Moreover, it is equal to the Dirichlet form of the function $q_{R}(\dot{\dot{N}})$. One thus has the bound

$$
\mathcal{E}_{N}\left(q_{R}, q_{R}\right) \leq c R^{d-2}
$$

Further, note that when $y^{\prime} \sim y$ in $\mathbb{L}_{N}$ (see below (4.9) for notation) are not both in $C_{R}$, $\left|\bar{\varphi}\left(y^{\prime}\right)-\bar{\varphi}(y)\right|^{2} \leq 2\left(\left|\psi\left(y^{\prime}\right)-\psi(y)\right|^{2}+a_{R}^{2}\left|q_{R}\left(y^{\prime}\right)-q_{R}(y)\right|^{2}+\left|\gamma\left(y^{\prime}\right)-\gamma(y)\right|^{2}+b_{R}^{2}\left|q_{R}\left(y^{\prime}\right)-q_{R}(y)\right|^{2}\right)$.

Thus, coming back to (5.25), we see that, denoting by $\sum^{\prime}$ the summation over $y^{\prime} \sim y$ in $\mathbb{L}_{N}$, not both in $C_{R}$, one has

$$
\begin{aligned}
\mathcal{E}_{N}(\bar{\varphi}, \bar{\varphi}) \leq & \mathcal{E}_{N}(\varphi, \varphi)+\frac{c}{N^{d-2}} \sum^{\prime}\left(\left|\psi\left(y^{\prime}\right)-\psi(y)\right|^{2}+\left|\gamma\left(y^{\prime}\right)-\gamma(y)\right|^{2}\right) \\
& +2\left(a_{R}^{2}+b_{R}^{2}\right) \mathcal{E}_{N}\left(q_{R}, q_{R}\right) \\
\stackrel{(5.24)}{\leq} & \mathcal{E}_{N}(\varphi, \varphi)+\left(\frac{c}{N^{d-2}} \sum_{\ell \geq N R} \frac{\ell^{d-1}}{\left(\frac{\ell}{N}\right)^{2(d-2)}} \frac{1}{\ell^{2}}+\frac{c}{R^{d-2}}\right)\left(\min _{\partial C_{0}} \psi^{2}+\min _{\partial C_{0}} \gamma^{2}\right) \\
\leq & \mathcal{E}_{N}(\varphi, \varphi)+\frac{c}{R^{d-2}}\left(\min _{\partial C_{0}} \psi^{2}+\min _{\partial C_{0}} \gamma^{2}\right) .
\end{aligned}
$$

Since the capacity of a finite subset of $\mathbb{Z}^{d}$ is smaller than the Dirichlet form of any function in the extended Dirichlet space equal to 1 on the set, and $\psi$, resp. $\gamma$, is bigger or equal to $\min _{\partial C_{0}} \psi$, resp. $\min _{\partial C_{0}} \gamma$, on $\left(\partial C_{0}\right) \cap \mathbb{L}_{N}$, we find that

$$
\mathcal{E}_{N}(\psi, \psi) \geq \frac{d}{N^{d-2}} \operatorname{cap}_{\mathbb{Z}^{d}}\left(N \partial C_{0}\right)\left(\min _{\partial C_{0}} \psi\right)^{2} \geq c\left(\min _{\partial C_{0}} \psi\right)^{2} \text { and likewise } \mathcal{E}_{N}(\gamma, \gamma) \geq c\left(\min _{\partial C_{0}} \gamma\right)^{2}
$$

Inserting these bounds in the last line of (5.27) and using (5.21), we find

$$
\mathcal{E}_{N}(\bar{\varphi}, \bar{\varphi}) \leq \mathcal{E}_{N}(\varphi, \varphi)\left(1+\frac{c}{R^{d-2}}\right)
$$

Moreover, by (5.25), $\bar{\varphi}=\varphi$ on $C_{R}$ and 0 on $\mathbb{L}_{N} \backslash C_{100 R}$. We have proved Lemma 5.7.

We resume the proof of (5.17). We denote by $\alpha \in[0, \infty]$ the left-hand side of $(5.17)$. Without loss of generality, we can assume $\alpha<\infty$, otherwise (5.17) is immediate. We consider a subsequence $N_{\ell}, \ell \geq 1$, as well as sequences $\mu_{\ell} \in M_{+}(B)$, and $\varphi_{\ell} \geq-1$ on $B_{N_{\ell}}$, of functions on $\mathbb{L}_{N_{\ell}}$ harmonic outside $B_{N_{\ell}}$ and tending to 0 at infinity, such that using (5.10) and (4.12)

$$
\begin{gathered}
\mu_{\ell} \in K, \text { and } I_{N_{\ell}}\left(\mu_{\ell}\right) \rightarrow \alpha\left(\stackrel{\text { def }}{=} \frac{\underline{\lim }}{N} \inf _{\rho \in K} I_{N}(\rho)\right), \\
\left\{\begin{aligned}
\text { i }) & \mu_{\ell}=\frac{1}{N_{\ell}^{d}} \sum_{y \in B_{N_{\ell}}}\left(1+\varphi_{\ell}(y)\right)^{2} \delta_{y} \\
\text { ii }) & I_{N_{\ell}}\left(\mu_{\ell}\right)=\mathcal{E}_{N_{\ell}}\left(\varphi_{\ell}, \varphi_{\ell}\right) .
\end{aligned}\right.
\end{gathered}
$$

We choose $R \geq 1$, and construct with the help of Lemma 5.7 a sequence $\bar{\varphi}_{\ell}, \ell \geq 1$, of functions on $\mathbb{L}_{N_{\ell}}$ for which (5.20) holds (with $\varphi$ replaced by $\varphi_{\ell}$ and $N$ by $N_{\ell}$ ). The 
functions $\bar{\varphi}_{\ell}$ vanish outside $C_{100 R} \cap \mathbb{L}_{N_{\ell}}$. Since the principal Dirichlet eigenvalue of the discrete Laplacian in a box of side-length $L \geq 1$ in $\mathbb{Z}^{d}$ is at least $c L^{-2}$, see for instance [14], p. 185, we find that

$$
\left\|\bar{\varphi}_{\ell}\right\|_{L^{2}\left(\mathbb{L}_{N_{\ell}}\right)} \leq c(B) R^{2} \mathcal{E}_{N_{\ell}}\left(\bar{\varphi}_{\ell}, \bar{\varphi}_{\ell}\right) \text { (and the limsup in } \ell \text { is at most } c^{\prime}(B) R^{2} \alpha \text { ). }
$$

The functions $\bar{\varphi}_{\ell}$ are defined on the different lattices $\mathbb{L}_{N_{\ell}}$, and it is convenient to introduce the functions $\Phi_{\ell}, \ell \geq 1$, on $\mathbb{R}^{d}$, which take the value $\bar{\varphi}_{\ell}(y)$ on $y+\frac{1}{N^{\ell}}[0,1)^{d}$, i.e.

$$
\Phi_{\ell}(z)=\sum_{y \in \mathbb{L}_{N_{\ell}}} \bar{\varphi}_{\ell}(y) 1_{y+\frac{1}{N_{\ell}}[0,1)^{d}}(z), z \in \mathbb{R}^{d}, \ell \geq 1 .
$$

Note that, by construction, for all $\ell \geq 1$,

$$
\begin{aligned}
& 1+\Phi_{\ell}(z) \geq 0, \text { if } z \in B \text { and } d_{\infty}(z, \partial B) \geq \frac{1}{N_{\ell}} \\
& \Phi_{\ell}=0, \text { on } \mathbb{R}^{d} \backslash C_{200 R}, \\
& \left\|\Phi_{\ell}\right\|_{L^{2}\left(\mathbb{R}^{d}\right)}=\left\|\bar{\varphi}_{\ell}\right\|_{L^{2}\left(\mathbb{L}_{N_{\ell}}\right)}\left(\text { and } \sup _{\ell}\left\|\Phi_{\ell}\right\|_{L^{2}\left(\mathbb{R}^{d}\right)}<\infty, \text { by }(5.30)\right) .
\end{aligned}
$$

We will now prove that the functions $\Phi_{\ell}, \ell \geq 1$, are equicontinuous in $L^{2}\left(\mathbb{R}^{d}\right)$ with respect to translations:

$$
\lim _{h \rightarrow 0} \sup _{\ell \geq 1}\left\|\Phi_{\ell}(\cdot+h)-\Phi_{\ell}(\cdot)\right\|_{L^{2}\left(\mathbb{R}^{d}\right)}=0
$$

Using the triangle inequality and translation invariance, we can assume, without loss of generality, that $h$ is parallel to and pointing in the direction of $e_{i}$, the $i$-th vector of the canonical basis of $\mathbb{R}^{d}$. For notational simplicity, we treat the case $i=1$ (the other cases are handled similarly).

We write $N_{\ell} h=(k+r) e_{1}$, where $k \geq 0$, is an integer and $0 \leq r<1$ (both depend on $\ell)$. We see that

$$
\begin{aligned}
& \left\|\Phi_{\ell}(\cdot+h)-\Phi_{\ell}(\cdot)\right\|_{L^{2}\left(\mathbb{R}^{d}\right)}^{2} \leq 2(a+b), \text { where } \\
& a=\left\|\Phi_{\ell}\left(\cdot+\frac{k}{N_{\ell}} e_{1}\right)-\Phi_{\ell}(\cdot)\right\|_{L^{2}\left(\mathbb{R}^{d}\right)}^{2} \text { and } b=\left\|\Phi_{\ell}\left(\cdot+\frac{r}{N_{\ell}} e_{1}\right)-\Phi_{\ell}(\cdot)\right\|_{L^{2}\left(\mathbb{R}^{d}\right)}^{2}
\end{aligned}
$$

Expressing $b$ in terms of $\bar{\varphi}_{\ell}$, we find that

$$
b=r\left\|\bar{\varphi}_{\ell}\left(\cdot+\frac{e_{1}}{N_{\ell}}\right)-\bar{\varphi}_{\ell}(\cdot)\right\|_{L^{2}\left(\mathbb{L}_{N_{\ell}}\right)}^{2} \stackrel{(4.9)}{\leq} c \frac{r}{N_{\ell}^{2}} \mathcal{E}_{N_{\ell}}\left(\bar{\varphi}_{\ell}, \bar{\varphi}_{\ell}\right) \leq \frac{c}{N_{\ell}^{2}}|h| \mathcal{E}_{N_{\ell}}\left(\bar{\varphi}_{\ell}, \bar{\varphi}_{\ell}\right) .
$$

On the other hand, by the triangle inequality and translation invariance, we have

$$
\begin{aligned}
& a \leq k^{2}\left\|\Phi_{\ell}\left(\cdot+\frac{e_{1}}{N_{\ell}}\right)-\Phi_{\ell}(\cdot)\right\|_{L^{2}\left(\mathbb{R}^{d}\right)}^{2}=k^{2}\left\|\bar{\varphi}_{\ell}\left(\cdot+\frac{e_{1}}{N_{\ell}}\right)-\bar{\varphi}_{\ell}(\cdot)\right\|_{L^{2}\left(\mathbb{L}_{N_{\ell}}\right)}^{2} \\
& \stackrel{(4.10)}{\leq} c \frac{k^{2}}{N_{\ell}^{2}} \mathcal{E}_{N_{\ell}}\left(\bar{\varphi}_{\ell}, \bar{\varphi}_{\ell}\right) \leq c|h|^{2} \mathcal{E}_{N_{\ell}}\left(\bar{\varphi}_{\ell}, \bar{\varphi}_{\ell}\right) .
\end{aligned}
$$


Combining the bounds on $a$ and $b$, we see that for $h=|h| e_{1}$,

$$
\left\|\Phi_{\ell}(\cdot+h)-\Phi_{\ell}(\cdot)\right\|_{L^{2}\left(\mathbb{R}^{d}\right)}^{2} \leq c|h|\left(|h| \vee \frac{1}{N_{\ell}}\right) \mathcal{E}_{N_{\ell}}\left(\bar{\varphi}_{\ell}, \bar{\varphi}_{\ell}\right), \text { for } \ell \geq 1
$$

The claim (5.35) now follows since $\sup _{\ell} \mathcal{E}_{N_{\ell}}\left(\bar{\varphi}_{\ell}, \bar{\varphi}_{\ell}\right)<\infty$.

By $(5.30),(5.33),(5.35)$, and Theorem 2.21, p. 31 of [1], we find that $\Phi_{\ell}, \ell \geq 1$, is a relatively compact subset of $L^{2}\left(\mathbb{R}^{d}\right)$.

Hence, up to extraction of a subsequence (which we still denote by $\Phi_{\ell}$ ), we can assume that

$$
\Phi_{\ell} \underset{\ell}{\longrightarrow} \Phi \text { in } L^{2}\left(\mathbb{R}^{d}\right)
$$

and by (5.32), (5.33), we see that

$$
1+\Phi \geq 0 \text { a.e. on } B \text { and } \Phi=0 \text { a.e. on } \mathbb{R}^{d} \backslash C_{200 R} \text {. }
$$

By (5.37), we see that

$$
\left(1+\Phi_{\ell}\right)^{2} \underset{\ell}{\longrightarrow}(1+\Phi)^{2} \text { in } L_{\mathrm{loc}}^{1}\left(\mathbb{R}^{d}\right)
$$

By uniform integrability, it follows that the integral of $\left(1+\Phi_{\ell}\right)^{2}$ over $\left\{z \in \mathbb{R}^{d} ; d_{\infty}(z, \partial B) \leq\right.$ $\left.\frac{1}{N_{\ell}}\right\}$ tends to 0 with $\ell$. In addition, since $\bar{\varphi}_{\ell}=\varphi_{\ell}$ on $C_{R} \supseteq\left\{z \in \mathbb{R}^{d} ; d_{\infty}(z, B) \leq 1\right\}$, cf. (5.19), we see by (5.29) i) and (5.31) that $\mu_{\ell}\left(B \cap\left\{z \in \mathbb{R}^{d} ; d_{\infty}(z, \partial B) \leq \frac{1}{N_{\ell}}\right\}\right) \underset{\ell}{\longrightarrow}$. Thus, letting $B_{N}^{\prime}$ stand for the $y \in \mathbb{L}_{N}$ such that $y+\frac{1}{N}[0,1)^{d} \subseteq B$, we find that for $V \in C(B)$,

$$
\left\langle\mu_{\ell}, V\right\rangle-\int_{B}\left(1+\Phi_{\ell}\right)^{2} V d z=\sum_{y \in B_{N_{\ell}}^{\prime}} \int_{y+\frac{1}{N_{\ell}}[0,1)^{d}}(V(y)-V(z))\left(1+\Phi_{\ell}(z)\right)^{2} d z+o(1), \text { as } \ell \rightarrow \infty .
$$

Moreover, the sum in the right-hand side tends to 0 with $\ell$, by uniform continuity of $V$ and (5.39). Hence, by (5.39), we see that $\mu_{\ell}\left(\in K\right.$, by (5.28)) converges in $M_{+}(B)$ to $(1+\Phi)^{2} m_{B}$. This shows that

$$
\mu=(1+\Phi)^{2} m_{B} \in K
$$

Moreover, by (5.20) iii), (5.28), (5.29) ii), we find that

$$
\alpha\left(1+\frac{c(B)}{R^{d-2}}\right) \geq \varlimsup_{\ell} \mathcal{E}_{N_{\ell}}\left(\bar{\varphi}_{\ell}, \bar{\varphi}_{\ell}\right)=\varlimsup_{\ell} \frac{1}{2} \sum_{j=1}^{d} N_{\ell}^{2}\left\|\bar{\varphi}_{\ell}\left(\cdot+\frac{e_{j}}{N_{\ell}}\right)-\bar{\varphi}_{\ell}(\cdot)\right\|_{L^{2}\left(\mathbb{L}_{N_{\ell}}\right)}^{2}
$$

and expressing this last quantity in terms of $\Phi_{\ell}$ and using the Fourier transform

$$
\begin{aligned}
& =\varlimsup_{\ell} \frac{1}{2} \sum_{j=1}^{d} \frac{1}{(2 \pi)^{d}} \int_{\mathbb{R}^{d}} N_{\ell}^{2}\left|e^{i \frac{\xi_{j}}{N_{\ell}}}-1\right|^{2}\left|\widehat{\Phi}_{\ell}(\xi)\right|^{2} d \xi \\
& \stackrel{\text { Fatou }}{\geq} \frac{1}{2(2 \pi)^{d}} \int_{\mathbb{R}^{d}}|\xi|^{2}|\widehat{\Phi}(\xi)|^{2} d \xi=\frac{1}{2} \int_{\mathbb{R}^{d}}|\nabla \Phi(z)|^{2} d z
\end{aligned}
$$


(extracting some subsequence along which $\widehat{\Phi}_{\ell}$ converges a.e. to $\widehat{\Phi}$, before the last inequality). We have thus shown that $\Phi \in H^{1}\left(\mathbb{R}^{d}\right)$ and

$$
\mathcal{E}(\Phi, \Phi) \leq \alpha\left(1+\frac{c(B)}{R^{d-2}}\right)
$$

Combined with (5.38), (5.40), this implies, see (3.4), that

$$
\inf _{K} I \leq \alpha\left(1+\frac{c(B)}{R^{d-2}}\right) \text {, for any } R>1 .
$$

Letting $R \rightarrow \infty$, we obtain Proposition 5.6.

We now come to the main theorem of this section, see $(0.1),(0.3)$ for notation.

Theorem 5.8. (The large deviation principle, $u>0$ )

As $N \rightarrow \infty$, the laws of $\rho_{N, u}$ on $M_{+}(B)$ satisfy a large deviation principle at speed $N^{d-2}$, with convex, good rate function $\frac{1}{d} I_{u}$.

Proof. Combining Theorems 5.4 and 5.5, it follows from Theorem 4.1.11, p. 120 of [10], that $\rho_{N, u}$ satisfies a weak large deviation principle at speed $N^{d-2}$, with rate function $\frac{1}{d} I_{u}$ (which is good and convex by (3.2), (3.5)).

In addition, one has exponential tightness for the laws of the $\rho_{N, u}$, due to the fact that (see (3.3) and (3.11) of [31]) for small $\lambda>0$,

$$
\begin{aligned}
& \varlimsup_{N} \frac{1}{N^{d-2}} \log \overline{\mathbb{E}}\left[\exp \left\{N^{d-2}\left\langle\rho_{N, u}, \lambda 1_{B}\right\rangle\right\}\right] \stackrel{(4.6)}{=} \underset{(4.7)}{=} \\
& \varlimsup_{N} \frac{1}{d} \log \overline{\mathbb{E}}\left[e^{\left\langle\mathcal{L}_{N, u}, d \lambda 1_{B}\right\rangle}\right]=u c(B, \lambda)<\infty,
\end{aligned}
$$

and the Chebyshev Inequality. The claim (5.44) now follows (see p. 8 of [10]).

\section{An application}

In this section, we apply the large deviation principle proved in the last section, see Theorem 5.8, and control the probability of existence of "high local density" regions insulating a given compact subset of $\mathbb{R}^{d}$. Our main results appear in Theorems 6.2 and 6.4. Extensions are discussed in Remark 6.5. We begin with some definitions and preliminary remarks.

We consider a compact set $K$ and a closed box $B_{0}$ in $\mathbb{R}^{d}$ such that

$$
\phi \neq K \subset B_{0} .
$$

Given $a \in \mathbb{R}$ and a continuous function $f$ on $\mathbb{R}^{d}$, we say that $\{f \geq a\}$ disconnects $K$ from $\partial B_{0}$, if

for any continuous function $\psi:[0,1] \rightarrow B_{0}$, such that $\psi(0) \in K$ and $\psi(1) \in \partial B_{0}$, one has $\sup _{0 \leq t \leq 1} f(\psi(t)) \geq a$. 
Note that the collection of bounded continuous functions $f$ for which $\{f \geq a\}$ disconnects $K$ from $\partial B_{0}$ is closed for the sup-norm topology.

Further, we consider $\delta \in(0,1)$, and a closed box $B$, so that

$$
K \subset B_{0} \subset B \text {, and } d\left(\partial B_{0}, \partial B\right)>\delta,
$$

as well as a continuous probability density $\varphi_{\delta}$ (with respect to Lebesgue measure), which is supported in $B(0, \delta)$. Denoting by $C_{0}\left(\mathbb{R}^{d}\right)$ the set of continuous functions on $\mathbb{R}^{d}$ that tend to 0 at infinity (endowed with the sup-norm topology), we consider the regularization map $r_{\delta}$ from $M_{+}(B)$ into $C_{0}\left(\mathbb{R}^{d}\right)$

$$
\mu \in M_{+}(B) \rightarrow r_{\delta}(\mu)(\cdot)=\int_{B} \varphi_{\delta}(\cdot-y) \mu(d y) \in C_{0}\left(\mathbb{R}^{d}\right)
$$

and introduce for $a \geq 0, \delta \in(0,1)$, the subset of $M_{+}(B)$

$$
\mathcal{D}_{a, \delta}=\left\{\mu \in M_{+}(B) ;\left\{r_{\delta}(\mu) \geq a\right\} \text { disconnects } K \text { from } \partial B_{0}\right\} .
$$

The next lemma collects some useful properties of the above objects.

Lemma 6.1. $(a \geq 0,0<\delta<1, u>0)$

(6.6) $\quad r_{\delta}$ is continuous.

(6.7) $\mathcal{D}_{a, \delta}$ is a closed subset of $M_{+}(B)$.

(6.8) The event $\left\{\rho_{N, u} \in \mathcal{D}_{a, \delta}\right\}$ does not depend on the choice of $B$ (satisfying (6.3)).

Proof. We start with the proof of (6.6). The functions $\varphi_{\delta}(z-\cdot)$ on $B$, as $z$ varies in $\mathbb{R}^{d}$, are equicontinuous at each point of $B$ and uniformly bounded. It now follows from Theorem 6.8, p. 51 of [19], that when $\mu_{n}$ converges weakly to $\mu$ in $M_{+}(B), r_{\delta}\left(\mu_{n}\right)$ converges uniformly to $r_{\delta}(\mu)$. This proves (6.6). Then, (6.7) is an immediate consequence of (6.6) and the observation below (6.2). As for (6.8), it suffices to notice that the restriction of $r_{\delta}\left(\rho_{N, u}\right)$ to $B_{0}$ does not depend on the choice of $B$ when (6.3) holds.

By the observation below (4.4) and (6.6), we know that for $u>0$, $\overline{\mathbb{P}}$-a.s., $r_{\delta}\left(\rho_{N, u}\right)$ converges uniformly to $r_{\delta}\left(u m_{B}\right)(\cdot)=u \int_{B} \varphi_{\delta}(\cdot-y) d y$, and this function equals $u$ on $B_{0}$ by (6.3). We will now consider the case where $a>u$ and study the large $N$ behavior of the probability of occurence of high values of $r_{\delta}\left(\rho_{N, u}\right)$ insulating $K$ from $\partial B_{0}$.

Theorem 6.2. (Insulation upper bound, $a>u>0$ )

$$
\varlimsup_{N} \frac{1}{N^{d-2}} \log \overline{\mathbb{P}}\left[\rho_{N, u} \in \mathcal{D}_{a, \delta}\right] \leq-\frac{1}{d}(\sqrt{a}-\sqrt{u})^{2} \operatorname{cap}(K)
$$

Proof. By (6.7) and Theorem 5.8 we have

$$
\varlimsup_{N} \frac{1}{N^{d-2}} \log \overline{\mathbb{P}}\left[\rho_{N, u} \in \mathcal{D}_{a, \delta}\right] \leq-\inf _{\mathcal{D}_{a, \delta}} \frac{1}{d} I_{u}
$$

We will use the notation $I_{u, B_{0}}$ or $I_{u, B}$ to highlight the dependence on the closed box at hand in the definition (0.3). We have the following control. 


\section{Lemma 6.3.}

$$
I_{u, B_{0}}\left(r_{\delta}(\mu) m_{B_{0}}\right) \leq I_{u, B}(\mu), \text { for any } \mu \in M_{+}(B)
$$

(we view $r_{\delta}(\mu) m_{B_{0}}$ as an element of $M_{+}\left(B_{0}\right)$ ).

Proof. Without loss of generality, we can assume that $I_{u, B}(\mu)<\infty$, so that $\mu=h m_{B}$ with $h \in L_{+}^{1}\left(m_{B}\right)$. Note that $y \in \mathbb{R}^{d} \rightarrow 1_{B_{0}}(\cdot) h(\cdot-y) \in L^{1}\left(m_{B_{0}}\right)$ is a continuous map (we extend $h$ outside $B$ as being equal to 0 ). Moreover, by (6.4)

$$
\left(1_{B_{0}} r_{\delta}(\mu)\right)(\cdot)=\int 1_{B_{0}}(\cdot) h(\cdot-y) \varphi_{\delta}(y) d y
$$

We also know that $f \in L_{+}^{1}\left(m_{B_{0}}\right) \rightarrow I_{u, B_{0}}\left(f m_{B_{0}}\right)$ is a convex, lower semi-continuous map, see (2.31), (3.1), (3.5). Hence, we have

$$
\begin{aligned}
& I_{u, B_{0}}\left(r_{\delta}(\mu) m_{B_{0}}\right) \leq \sup _{|y| \leq \delta} I_{u, B_{0}}\left(1_{B_{0}}(\cdot) h(\cdot-y) m_{B_{0}}\right) \\
& \stackrel{(3.1)}{=} \sup _{|y| \leq \delta} I_{u, B_{0}-y}\left(1_{B_{0}-y} h m_{B_{0}-y}\right) \\
& \underset{(6.3)}{\stackrel{(3.3)}{\leq}} I_{u, B}\left(h m_{B}\right)=I_{u, B}(\mu)
\end{aligned}
$$

This proves (6.11).

We will now bound the right-hand side of (6.10). Given $\mu \in \mathcal{D}_{a, \delta}$, we define

$$
\varphi(z)=E_{z}\left[\left(\sqrt{r_{\delta}(\mu)}-\sqrt{u}\right)\left(X_{H_{B_{0}}}\right), H_{B_{0}}<\infty\right], z \in \mathbb{R}^{d},
$$

so that $\varphi \in C_{0}\left(\mathbb{R}^{d}\right)$. If $I_{u, B_{0}}\left(r_{\delta}(\mu) m_{B_{0}}\right)<\infty$, then by (3.1), (2.31), and the explanation below (2.26), we have $\varphi \in \mathcal{F}_{e}$ and

$$
\mathcal{E}(\varphi, \varphi)=\widetilde{\mathcal{E}}_{B_{0}}\left(\sqrt{r_{\delta}(\mu)}-\sqrt{u}, \sqrt{r_{\delta}(\mu)}-\sqrt{u}\right) \underset{(3.5),(2.31)}{\stackrel{(3.1)}{=}} I_{u, B_{0}}\left(r_{\delta}(\mu) m_{B_{0}}\right) .
$$

In addition, $\varphi=\sqrt{r_{\delta}(\mu)}-\sqrt{u}$ on $B_{0}$, and since $\mu \in \mathcal{D}_{a, \delta}$, we see that $\{\varphi \geq \sqrt{a}-\sqrt{u}\}$ disconnects $K$ from $\partial B_{0}$. Setting $\widetilde{K}=\left\{y \in B_{0}, \varphi(y) \geq \sqrt{a}-\sqrt{u}\right\}$, it follows from Theorem 1.10, p. 58 of [20], that $\operatorname{cap}(\widetilde{K}) \geq \operatorname{cap}(K)$, and from p. 71 of [13], that $\mathcal{E}(\varphi, \varphi) \geq$ $(\sqrt{a}-\sqrt{u})^{2} \operatorname{cap}(\widetilde{K})$. Hence, for $\mu \in \mathcal{D}_{a, \delta}$, we have

$$
I_{u}(\mu) \stackrel{(6.11),(6.14)}{\geq} \mathcal{E}(\varphi, \varphi) \geq(\sqrt{a}-\sqrt{u})^{2} \operatorname{cap}(\widetilde{K}) \geq(\sqrt{a}-\sqrt{u})^{2} \operatorname{cap}(K) .
$$

Inserting this bound in the right-hand side of (6.10), we obtain (6.9).

We now complement the asymptotic upper bound from Theorem 6.2 with an asymptotic lower bound. We denote by $K^{\delta}=\left\{z \in \mathbb{R}^{d} ; d(z, K) \leq \delta\right\}$ the closed $\delta$-neighborhood of $K$ for the Euclidean distance. One knows that $\operatorname{cap}\left(K^{\delta}\right) \downarrow \operatorname{cap}(K)$ as $\delta \rightarrow 0$, see Remark 6.51 ) below. 
Theorem 6.4. (Insulation lower bound, $a>u>0$ )

$$
\frac{\lim }{N} \frac{1}{N^{d-2}} \log \overline{\mathbb{P}}\left[\rho_{N, u} \in \mathcal{D}_{a, \delta}\right] \geq-\frac{1}{d}(\sqrt{a}-\sqrt{u})^{2} \operatorname{cap}\left(K^{\delta}\right) .
$$

Proof. We consider $\varepsilon>0$ and define

$$
\mu_{\varepsilon}=(\sqrt{u}+(\sqrt{a+\varepsilon}-\sqrt{u}) h)^{2} m_{B},
$$

where $h(y)=P_{y}\left[\widetilde{H}_{K^{\delta}}<\infty\right]=P_{y}\left[H_{K^{\delta}}<\infty\right]$, for $y \in \mathbb{R}^{d}$, is the equilibrium potential of $K^{\delta}$ (every point of $K^{\delta}$ is regular for $K^{\delta}$ so $h \in C_{0}\left(\mathbb{R}^{d}\right)$ and $h=1$ on $K^{\delta}$ ). Observe that

$$
\begin{aligned}
r_{\delta}\left(\mu_{\varepsilon}\right)(y) \stackrel{(6.4)}{=} & \int_{B}(\sqrt{u}+(\sqrt{a+\varepsilon}-\sqrt{u}) h(z))^{2} \varphi_{\delta}(y-z) d z \\
& \geq a+\varepsilon, \text { when } y \in K
\end{aligned}
$$

(since $h=1$ on $K^{\delta}$, and this is actually an equality).

By (6.6), we can find an open neighborhood $O$ of $\mu_{\varepsilon}$ in $M_{+}(B)$ so that for all $\mu \in O$, $r_{\delta}(\mu) \geq a+\frac{\varepsilon}{2}$ on $K$. It follows that $\left\{r_{\delta}(\mu) \geq a\right\}$ separates $K$ from $\partial B_{0}$, for all $\mu \in O$, i.e. $O \subseteq \mathcal{D}_{a, \delta}$. As a result of Theorem 5.8 we find that

$$
\frac{\lim }{N} \frac{1}{N^{d-2}} \log \overline{\mathbb{P}}\left[\rho_{N, u} \in \mathcal{D}_{a, \delta}\right] \geq-\inf _{O} \frac{1}{d} I_{u} \geq-\frac{1}{d} I_{u}\left(\mu_{\varepsilon}\right) .
$$

Now $h$ belongs to $\mathcal{F}_{e}$ and $\mathcal{E}(h, h)=\operatorname{cap}\left(K^{\delta}\right)$, see [13], p. 71. Therefore, by (3.4), (3.5) and below (2.26),

$$
I_{u}\left(\mu_{\varepsilon}\right)=(\sqrt{a+\varepsilon}-\sqrt{u})^{2} \mathcal{E}(h, h)=(\sqrt{a+\varepsilon}-\sqrt{u})^{2} \operatorname{cap}\left(K^{\delta}\right) .
$$

Inserting this identity in (6.19) and letting $\varepsilon \rightarrow 0$, we obtain (6.16).

\section{Remark 6.5.}

1) One knows from Proposition 1.13, p. 60 of [20], that $\operatorname{cap}\left(K^{\delta}\right) \downarrow \operatorname{cap}(K)$, as $\delta \rightarrow 0$. So, when $\delta \rightarrow 0$, the constant in the right-hand side of the lower bound (6.16) tends to the constant in the right-hand side of the upper bound (6.9).

2) Actually, one can let $\delta$ slowly tend to 0 in (6.9) and (6.16). More precisely, given a choice of $\varphi_{\delta}$ for each $\delta \in(0,1), a>u>0$ and $K, B_{0}, B$ satisfying (6.3) when $\delta=\frac{1}{2}$, one can, using a diagonal type procedure, the remark above, and Theorems $6.2,6.4$, to construct a sequence $\delta_{N}$ slowly tending to zero so that

$$
\lim _{N} \frac{1}{N^{d-2}} \log \overline{\mathbb{P}}\left[\rho_{N, u} \in \mathcal{D}_{a, \delta_{N}}\right]=-\frac{1}{d}(\sqrt{a}-\sqrt{u})^{2} \operatorname{cap}(K) .
$$

3) We can, in place of $\mathcal{D}_{a, \delta}$ in (6.5), instead consider "disconnection by sub-level sets", i.e.

$$
\mathcal{D}_{a, \delta}^{\prime}=\left\{\mu \in M_{+}(B) ;\left\{r_{\delta}(\mu) \leq a\right\} \text { disconnects } K \text { from } \partial B_{0}\right\}
$$

(replacing $\sup _{0 \leq t \leq 1} f(\psi(t)) \geq a$ by $\inf _{0 \leq t \leq 1} f(\psi(t)) \leq a$ in (6.2) to define the above event). 
The same arguments of Theorems 6.2 and 6.4 apply (one replaces $a+\varepsilon$ by $a-\varepsilon$, in (6.17), with $0<\varepsilon<a$ ) and we obtain that for $0<a<u, 0<\delta<1$, under (6.3)

$$
\begin{aligned}
& \varlimsup_{N} \frac{1}{N^{d-2}} \log \overline{\mathbb{P}}\left[\rho_{N, u} \in \mathcal{D}_{a, \delta}^{\prime}\right] \leq-\frac{1}{d}(\sqrt{a}-\sqrt{u})^{2} \operatorname{cap}(K), \\
& \varlimsup_{N} \frac{1}{N^{d-2}} \log \overline{\mathbb{P}}\left[\rho_{N, u} \in \mathcal{D}_{a, \delta}^{\prime}\right] \geq-\frac{1}{d}(\sqrt{a}-\sqrt{u})^{2} \operatorname{cap}\left(K^{\delta}\right),
\end{aligned}
$$

4) It is instructive to compare (6.16) in Theorem 6.4 with the lower bound one obtains by the following intuitive "change of measure" strategy. Namely, assume $0<u<a$ and introduce the new probability measure

$$
\widetilde{\mathbb{P}}_{N}=e^{\lambda \eta-u \operatorname{cap}_{\mathbb{Z}^{d}}\left(K^{\delta, N}\right)\left(e^{\lambda}-1\right)} \overline{\mathbb{P}}
$$

where $\eta$ stands for the total number of bilateral trajectories modulo time-shift with label at most $u$, which enter $K^{\delta, N}=\left(N K^{\delta}\right) \cap \mathbb{Z}^{d}, \lambda=\log \left(\frac{a+\varepsilon}{u}\right)$, and we recall that for $A$ finite in $\mathbb{Z}^{d}, \operatorname{cap}_{\mathbb{Z}^{d}}(A)$ stands for the capacity of $A$ (attached to the simple random walk on $\mathbb{Z}^{d}$ ).

Under $\widetilde{\mathbb{P}}_{N}$, the variable $\eta$ has Poisson distribution with parameter $(a+\varepsilon) \operatorname{cap}_{\mathbb{Z}^{d}}\left(K^{\delta, N}\right)$ (instead of $u \operatorname{cap}_{\mathbb{Z}^{d}}\left(K^{\delta, N}\right)$ under $\left.\overline{\mathbb{P}}\right)$, and $\left(L_{x, u}\right)_{x \in K^{\delta, N}}$ has the same distribution as $\left(L_{x, a+\varepsilon}\right)_{x \in K^{\delta, N}}$ under $\overline{\mathbb{P}}$. As we now explain,

$$
\lim _{N} \widetilde{\mathbb{P}}_{N}\left(\rho_{N, u} \in \mathcal{D}_{a, \delta}\right)=1
$$

Indeed, by (4.4), $\overline{\mathbb{P}}$-a.s., $\frac{1}{N^{d}} \sum_{y \in \mathbb{L}_{N}} L_{N y, a+\varepsilon} \delta_{y}$ converges vaguely to $(a+\varepsilon) d y$, as $N \rightarrow \infty$, and the restriction to $K$ of $r_{\delta}\left(\rho_{N, u}\right)$ under $\widetilde{\mathbb{P}}_{N}$ has the same distribution as the restriction to $K$ of $r_{\delta}\left(\rho_{N, a+\varepsilon}\right)$ under $\overline{\mathbb{P}}$, which $\overline{\mathbb{P}}$-a.s. converges uniformly on $K$ to $a+\varepsilon$. However, as soon as the restriction to $K$ of $r_{\delta}\left(\rho_{N, u}\right)$ exceeds $a$ everywhere, the event $\left\{\rho_{N, u} \in \mathcal{D}_{a, \delta}\right\}$ occurs, and (6.24) follows.

By the classical relative entropy estimate, see for instance [11], p. 76, and (6.24),

$$
\liminf _{N} \frac{1}{N^{d-2}} \log \overline{\mathbb{P}}\left[\rho_{N, u} \in \mathcal{D}_{a, \delta}\right] \geq-\limsup _{N} \frac{1}{N^{d-2}} H\left(\widetilde{\mathbb{P}}_{N} \mid \overline{\mathbb{P}}\right),
$$

where $H\left(\widetilde{\mathbb{P}}_{N} \mid \overline{\mathbb{P}}\right)=\mathbb{E}^{\widetilde{\mathbb{P}}_{N}}\left[\log \frac{d \widetilde{\mathbb{P}}_{N}}{d \overline{\mathbb{P}}}\right]$ stands for the relative entropy of $\widetilde{\mathbb{P}}_{N}$ with respect to $\overline{\mathbb{P}}$. As we now explain,

$$
\liminf _{N} \frac{1}{N^{d-2}} H\left(\widetilde{\mathbb{P}}_{N} \mid \overline{\mathbb{P}}\right) \geq\left(a \log \frac{a}{u}-a+u\right) \frac{1}{d} \operatorname{cap}\left(K^{\delta}\right),
$$

and since (as can be checked directly)

$$
v \log \frac{v}{u}-v+u>(\sqrt{v}-\sqrt{u})^{2} \text {, for any } v, u>0 \text {, with } v \neq u,
$$

the lower bound (6.25) is worse than (6.16).

To prove $(6.26)$, one notes that by our choice of $\widetilde{\mathbb{P}}_{N}$,

$$
\begin{aligned}
H\left(\widetilde{\mathbb{P}}_{N} \mid \overline{\mathbb{P}}\right) & =E^{\widetilde{\mathbb{P}}_{N}}[\lambda \eta]-(a+\varepsilon-u) \operatorname{cap}_{\mathbb{Z}^{d}}\left(K^{\delta, N}\right) \\
& =\left((a+\varepsilon) \log \left(\frac{a+\varepsilon}{u}\right)-(a+\varepsilon)+u\right) \operatorname{cap}_{\mathbb{Z}^{d}}\left(K^{\delta, N}\right) .
\end{aligned}
$$


The same argument leading to (5.37), (5.42) shows that for each $R \geq 1$ and $\delta^{\prime} \in(0, \delta)$ one can construct $\Phi \in H^{1}\left(\mathbb{R}^{d}\right)$ with compact support, which is a.e. equal to 1 on $K^{\delta^{\prime}}$, and such that $\mathcal{E}(\Phi, \Phi) \leq d\left(1+\frac{c(B)}{R^{d-2}}\right) \beta$ with $\beta=\underline{\lim }_{N} \frac{1}{N^{d-2}} \operatorname{cap}_{\mathbb{Z}^{d}}\left(K^{\delta, N}\right)$. It thus follows (see [13], p. 71) that

$$
\beta \geq \frac{1}{d}\left(1+\frac{c(B)}{R^{d-2}}\right)^{-1} \operatorname{cap}\left(K^{\delta^{\prime}}\right) .
$$

Letting $R \rightarrow \infty, \delta^{\prime} \uparrow \delta$, and noting that when $y \in K^{\delta}, P_{y^{-}}$a.s. Brownian motion immediately hits $\bigcup_{\delta^{\prime}<\delta} K^{\delta^{\prime}}$, so that by Theorem 1.10 and Proposition 1.13, p. 58 and 60 of [20], $\operatorname{cap}\left(K^{\delta^{\prime}}\right) \uparrow \operatorname{cap}\left(K^{\delta}\right)$ as $\delta^{\prime} \uparrow \delta$, we obtain that

$$
\liminf _{N} \frac{1}{N^{d-2}} \operatorname{cap}_{\mathbb{Z}^{d}}\left(K^{\delta, N}\right) \geq \frac{1}{d} \operatorname{cap}\left(K^{\delta}\right) .
$$

Since $v \rightarrow v \log \frac{v}{u}-v+u$ is increasing for $v \in(u, \infty)$, the combination of (6.28) and (6.29) readily yields $(6.26)$.

So, the intuitive lower bound we just described does not capture (6.16).

5) It is an important feature of random interlacements that the vacant set $\mathcal{V}^{u}$ of random interlacements at level $u$ on $\mathbb{Z}^{d}, d \geq 3$, undergoes a phase transition from a percolative regime, when $u<u_{*}$, to a non-percolative regime, when $u>u_{*}$, with $u_{*}$ a certain nondegenerate critical value, which is positive and finite (see [28], [25], and also [12], [22] for recent developments). Given a smooth compact subset $K$ of $\mathbb{R}^{d}$ and its discrete blow-up $K^{N}=(N K) \cap \mathbb{Z}^{d}$, one can consider the disconnection event $\left\{K^{N} \stackrel{\mathcal{V}^{u}}{\longleftrightarrow} \infty\right\}$, for which the connected components of $K^{N} \cap \mathcal{V}^{u}$ in $\mathcal{V}^{u}$ are finite (possibly empty). Looking at a small interior ball in $K$ and its discrete blow-up, it is straightforward to argue that for $u<u_{*}, \lim _{N} \overline{\mathbb{P}}\left[K^{N} \stackrel{\mathcal{V}^{u}}{\longleftrightarrow} \infty\right]=0$. One can wonder whether the main effect in realizing this atypical disconnection event stems from a large deviation of the density profile of occupation-times, for which, roughly speaking, values of the profile exceeding $u_{*}$ would insulate $K$ from infinity, and whether one has the asymptotics

$$
\lim _{N} \frac{1}{N^{d-2}} \log \overline{\mathbb{P}}\left[K^{N} \stackrel{\mathcal{V}^{u}}{\longleftrightarrow} \infty\right]=-\frac{1}{d}\left(\sqrt{u}_{*}-\sqrt{u}\right)^{2} \operatorname{cap}(K), \text { for } u<u_{*} .
$$

We refer to [18], for a lower bound on the left-hand side of a similar nature.

\section{References}

[1] R.A. Adams. Sobolev Spaces. Academic Press, New York, 1975.

[2] P. Billingsley. Convergence of probability measures. Wiley, New York, 1968.

[3] E. Bolthausen and J.D. Deuschel. Critical large deviations for Gaussian fields in the phase transition regime, I. Ann. Probab., 21(4):1876-1920, 1993.

[4] R. Cerf. Large deviations for three dimensional supercritical percolation. Astérisque 267, Société Mathématique de France, 2000. 
[5] J. Černý, A. Teixeira, and D. Windisch. Giant vacant component left by a random walk in a random $d$-regular graph. Ann. Inst. Henri Poincaré Probab. Stat., 47(4):929968, 2011.

[6] Z.-Q. Chen. Gaugeability and conditional gaugeability. Transactions of the AMS, 354(11):4639-4679, 2002.

[7] Z.-Q. Chen and R. Song. General Gauge and conditional Gauge theorems. Ann. Probab., 30(3):1313-1339, 2002.

[8] K.L. Chung and Z. Zhao. From Brownian motion to Schrödinger's equation. Springer, New York, 1995.

[9] G. Dal Maso. An introduction to Г-convergence. Birkhäuser, Basel, 1993.

[10] A. Dembo and O. Zeitouni. Large deviations techniques and applications. Springer, Berlin, 2nd edition, 1998.

[11] J.D. Deuschel and D.W. Stroock. Large deviations. Academic Press, Boston, 1989.

[12] A. Drewitz, B. Ráth, and A. Sapozhnikov. Local percolative properties of the vacant set of random interlacements with small intensity. To appear in Annales de l'Institut Henri Poincaré, Probabilités et Statistiques, also available at arXiv:1206.6635, 2012.

[13] M. Fukushima, Y. Oshima, and M. Takeda. Dirichlet forms and symmetric Markov processes. Walter de Gruyter, Berlin, 1994.

[14] J. Glimm and A. Jaffe. Quantum Physics. Springer, Berlin, 1981.

[15] G. Grimmett. Percolation. Second edition, Springer, Berlin, 1999.

[16] U. Krengel. Ergodic theorems. Walter de Gruyter, Berlin, 1985.

[17] G.F. Lawler. Intersections of random walks. Birkhäuser, Basel, 1991.

[18] X. Li and A.S. Sznitman. A lower bound for disconnection by random interlacements. Electron. J. Probab., 19(17):1-26, 2014.

[19] K.R. Parthasarathy. Probability measures on metric spaces. Academic Press, New York, 1967.

[20] S.C. Port and C.J. Stone. Brownian motion and classical potential theory. Academic Press, New York, 1978.

[21] R.G. Pinsky. Positive harmonic functions and diffusion. Cambridge University Press, Cambridge, 1995.

[22] S. Popov and A. Teixeira. Soft local times and decoupling of random interlacements. To appear in J. Eur. Math. Soc., also available at arXiv: 1212.1605.

[23] S.I. Resnick. Extreme Values, regular variation, and point processes. Springer, New York, 1987. 
[24] W. Rudin. Functional analysis. Tata Mc Graw-Hill, New Delhi, 1974.

[25] V. Sidoravicius and A.S. Sznitman. Percolation for the vacant set of random interlacements. Comm. Pure Appl. Math., 62(6):831-858, 2009.

[26] A.S. Sznitman. Brownian motion, obstacles and random media. Springer, Berlin, 1998.

[27] A.S. Sznitman. On the domination of random walk on a discrete cylinder by random interlacements. Electron. J. Probab., 14:1670-1704, 2009.

[28] A.S. Sznitman. Vacant set of random interlacements and percolation. Ann. Math., 171:2039-2087, 2010.

[29] A.S. Sznitman. Random interlacements and the Gaussian free field. Ann. Probab., 40(6):2400-2438, 2012.

[30] A.S. Sznitman. An isomorphism theorem for random interlacements. Electron. Commun. Probab., 17(9):1-9, 2012.

[31] A.S. Sznitman. On scaling limits and Brownian interlacements. Bull. Braz. Math. Soc., New Series, 44(4) 555-592, 2013. Special issue IMPA 60 years.

[32] A. Teixeira and D. Windisch. On the fragmentation of a torus by random walk. Commun. Pure Appl. Math., 64(12):1599-1646, 2011. 